\title{
Impacts of surface gravity waves on a tidal front: A coupled model perspective
}

\author{
Brumer Sophia ${ }^{3,}{ }^{*}$, Garnier Valerie ${ }^{1}$, Redelsperger Jean-Luc ${ }^{3}$, Bouin Marie-Noelle ${ }^{2,3}$, \\ Ardhuin Fabrice ${ }^{3}$, Accensi Mickael ${ }^{1}$
}

\begin{abstract}
${ }^{1}$ Laboratoire d'Océanographie Physique et Spatiale, UMR 6523 IFREMER-CNRS-IRD-UBO, IUEM, Ifremer, ZI Pointe du Diable, CS10070, 29280, Plouzané, France

2 CNRM-Météo-France, 42 av. G. Coriolis, 31000 Toulouse, France

${ }^{3}$ Laboratoire d'Océanographie Physique et Spatiale, UMR 6523 IFREMER-CNRS-IRD-UBO, IUEM, Ifremer, ZI Pointe du Diable, CS10070, 29280, Plouzané, France
\end{abstract}

*Corresponding author : Sophia Brumer, email address : sophia.brumer@ifremer.fr

\begin{abstract}
:
A set of realistic coastal coupled ocean-wave numerical simulations is used to study the impact of surface gravity waves on a tidal temperature front and surface currents. The processes at play are elucidated through analyses of the budgets of the horizontal momentum, the temperature, and the turbulence closure equations. The numerical system consists of a 3D coastal hydrodynamic circulation model (Model for Applications at Regional Scale, MARS3D) and the third generation wave model WAVEWATCH III (WW3) coupled with OASIS-MCT at horizontal resolutions of 500 and $1500 \mathrm{~m}$, respectively. The models were run for a period of low to moderate southwesterly winds as observed during the Front de Marée Variable (FroMVar) field campaign in the Iroise Sea where a seasonal small-scale tidal sea surface temperature front is present. Over the 2 day period considered, long fetch waves grow gradually propagating north east and east.
\end{abstract}

Contrasting a stand-alone ocean run with a coupled ocean-wave run shows that waves move the Ushant front offshore by up to 4 kilometres and cool the offshore stratified side of the front by up to $1.5^{\circ} \mathrm{C}$. The analysis of the temperature budget shows that the change in advection is the dominant factor contributing to the frontal shift while the contribution of wave enhanced vertical temperature diffusion is secondary. Temperature, considered to be a tracer, is advected in the coupled run by the Lagrangian current resulting from the quasi-Eulerian and Stokes drift. Although the Stokes drift is directed shorewards, changes in the quasi-Eulerian current lead to a more offshore advection in the coupled than the stand-alone run. The quasi-Eulerian current is reduced (enhanced) during the ebb (flood) flow which correspond to periods of wave-following (-opposing) currents. This is due to wave breaking enhanced vertical mixing acting on the positive vertical gradient present in the quasi-Eulerian current during both ebb and flood tides. Partially coupled runs reveal that it is the surface flux of TKE associated to wave breaking that is key rather than the changes in the surface stress. They further elucidate the role of other modelled wave related processes. Although the contribution of the Stokes-Coriolis force and the wave breaking induced enhancement in vertical mixing to the quasi-Eulerian current are of similar magnitude and sign, it does not contribute significantly to the frontal shift. This is because it partially counters the Stokes drift advection which pushes the front shorewards. All Stokes drift related processes combined thus only lead to a very slight displacement of the front. 


\section{Highlights}

- A novel ocean-wave coupled modelling and analysis framework was developed. Realistic high resolution numerical simulations reveal wave-current interactions. Wave enhanced mixing reduces or enhances surface currents when following or opposing. Waves alter the position of the surface temperature tidal front in the Iroise Sea. Wave-ocean dynamics and kinematics in a tidally driven coastal ocean are described.

Keywords : Ocean-wave coupling, Coastal coupled modelling, Current-wave interactions, Tidal front, Iroise Sea 
In recent years much effort has been dedicated to understanding and quantifying the impacts of surface gravity waves on upper ocean currents and mixing as these can have significant physical and biogeochemical consequences (see Sullivan and McWilliams, 2010; Cavaleri et al., 2012; Villas Bô̂s et al., 2019, and references therein). The impacts in coastal seas are particularly relevant to a wide range of scientific questions and applications (Cavaleri et al., 2018). Modeling wave-current interactions in coastal environments presents many challenges since they are governed by the interplay of multiple phenomena spanning a wide range of spatio-temporal scales.

Waves alter the momentum and energy input to the upper ocean. They take up a portion of the wind input as they grow and release some to the ocean as they break. They generate a mean Lagrangian current in the direction of wave propagation known as the Stokes drift. On the rotating earth, the Stokes drift is affected by the Coriolis force inducing a current perpendicular to wave propagation and a transport that compensates the Stokes drift over an inertial period (Hasselmann, 1970; Xu and Bowen, 1994). Interaction between the Stokes drift velocity and the mean-flow vorticity results in the Stokes vortex force (Craik and Leibovich, 1976; Lane et al., 2007). The persistent vertical shear of the Stokes drift tilts the vertical component of the vorticity that is perpendicular to the wind into the wind direction. This promotes the generation of rolls aligned with the wind known as Langmuir circulations (Langmuir, 1938). A thorough overview of the effects of the Stokes drift on currents can be found in Suzuki and Fox-Kemper (2016). Wave breaking, Langmuir circulations, Stokes drift interaction with the Coriolis force, and stirring by non-breaking waves all contribute to upper-ocean mixing and turbulence ( Wu et al., 2015) as shown from measurements of the dissipation of the turbulent kinetic energy (Agrawal et al., 1992; Terray et al., 1996).

A common and highly predictable feature of coastal oceans are tidal fronts. These surface temperature fronts separate cold shallow waters nearshore where tidal friction produces enough turbulence to constantly mix the entire water column and warm waters offshore over depths where tidal mixing is not sufficient to erode the seasonal thermocline. They appear in the spring and erode at the end of autumn. Such fronts present convergence zones associated with wave steepening and breaking (Baschek et al., 2006). How in turn waves alter ocean currents, temperatures, and mixing around tidal fronts has not been studied in great detail because wave forcing is secondary to tidal forcing. The wave adjustement of geostrophic fronts was studied by McWilliams and Fox-Kemper (2013) and Suzuki et al. (2016) showed the importance of the Stokes shear in frontogenesis as they found it to energise the ageostrophic secondary circulations. In these studies, the Stokes drift is of the same order of magnitude as the frontal circulation and both 
are of the order or larger than the background flow. This is not the case around tidal fronts where the background tidal flow can be an order of magnitude larger than the frontal circulation and the Stokes drift. The set up of tidal fronts depends on the competing stratifying effect of solar radiation and tide driven mixing. Their location is largely determined by the magnitude of the tidal current and the water depth (Simpson, 1981). However, wave driven currents and mixing could alter the frontal position as this paper aims to elucidate.

Distinguishing wave effects in current measurements is not a straightforward exercise (Polton et al., 2005; Rascle and Ardhuin, 2009; Ardhuin et al., 2009) and often does not allow for much more than qualitative inferences. Laboratory experiments (Kemp and Simons, 1982, 1983; Klopman, 1993, 1994) provided insights on how surface waves alter currents. The dynamics they imply have yet to be verified in the field which may be in a different parameter regime and their existence in the real or modeled ocean needs to be assessed. Theoretical frameworks provide insights into key physical processes but the high complexity of the system does not allow to go much beyond asymptotic behaviour (McWilliams et al., 2004) and specific equilibria (McWilliams and Fox-Kemper, 2013; Song, 2009). Numerical methods allow to better understand the processes at play. However, asymptotic approaches and parameterisations remain necessary in models. One dimensional (vertical) models have been used to study wave modification to the Ekman current trying to reproduce in situ current shear measurements (Rascle et al., 2006; Rascle and Ardhuin, 2009). Large eddy simulations (Polton et al., 2005; McWilliams and Fox-Kemper, 2013; Sullivan et al., 2007; Suzuki et al., 2016) and direct numerical simulations (Sullivan et al., 2004) remain idealistic and only represent a subset of wave related processes.

To investigate the multitude and complexity of processes at play in the coastal oceans, realistic fullycoupled ocean-wave simulations are needed. In coupled modeling, the inclusion of wave processes heavily relies on parameterizations. Also, the representation of wave-current interactions is simpler than in the above mentioned theoretical work. They are, however, largely based on assumptions supported by those 'idealised' approaches and coupled simulations can provide a broader view of the processes at play. To date, several coupled model frameworks have been developed and exploited to study the impact of waves on currents in coastal seas (Osuna and Wolf, 2005; Bolaños et al., 2014; Ràfols et al., 2019). While Bolaños et al. (2014) concluded that waves do not significantly alter the tidally dominated component of the current but improved the transverse current component, Osuna and Wolf (2005) showed large effects of waves on currents especially where the currents are strong and sheared. Ràfols et al. (2019) reported no substantial differences in the water current field between coupled and uncoupled simulations.

It is important to note here that to date the majority of studies, be it theoretical, numerical, or 
experimental, have focused on cases where the Stokes drift is comparable or stronger than the background flow. Filling this gap, a new set of coupled model experiments were undertaken focusing on the Iroise Sea which provides an excellent natural laboratory to study interactions between the waves, the ocean, and the atmosphere in strongly tidally forced conditions. Located in the North Atlantic, off the coast of Brittany, France, the Iroise Sea is subject to long fetch waves and features an intense seasonal surface thermal front: the Ushant tidal front (Fig. 1). It is characterized by a strong surface temperature gradient separating the homogeneous waters of moderate temperatures $\left(<14^{\circ} \mathrm{C}\right)$ close to the coast and the warm surface $\left(>16^{\circ} \mathrm{C}\right)$ stratified waters offshore. A second temperature front exists at the bottom separating the waters of the cold "tongue" which covers the continental shelf and the homogeneous waters close to the coast. Le Boyer et al. (2009) noted that the two fronts are governed by different dynamics meaning that the two fronts are not always co-located. The position of the surface front varies with the tidal current. It is pushed towards the coast during the flood tide and towards the open sea by the ebb tide.

This paper provides a detailed study of the wave-ocean coupling in the Iroise Sea with a focus on the impacts on the currents and surface temperatures around the Ushant front based on a set of realistic forced and coupled coastal model runs. These are described in Section 2 along with an outline of the coupling framework. Key results are presented in Section 3 and discussed in Section 4. The conclusion section summarises the results and processes at play. Finally, it highlights the interest of the proposed model and analysis framework.

\section{Model Experiments}

In this section, the equations at the heart of the stand-alone ocean model are presented first followed by those used in the coupled configuration. Relevant aspects of the coupling between the ocean and the wave models are detailed exposing how wave related processes are taken into account. These include Stokes drift advection of tracers, Stokes-Coriolis and Stokes vortex forces on the horizontal currents, wave altered surface stress, wave breaking induced turbulence, and wave orbital velocity driven bottom stress. Configurations used for the ocean and wave models are then summarized along with the description of the simulations' setup. The equations of the wave model with currents are not recalled herein, as the focus of the paper is on the ocean response to waves. These can be found in Ardhuin et al. (2017). 
The ocean model used in this study is the 3D coastal hydrodynamic circulation model MARS3D (Model for Applications at Regional Scale, Lazure and Dumas (2008); Lazure et al. (2009)). MARS3D resolves the primitive equations for an in-compressible, hydrostatic fluid under the Boussinesq hypothesis. The temporal evolution of a horizontal current $(\mathbf{u}=u+i v$ in $\mathbf{x}=x+i y)$ is modeled as:

$$
\frac{\partial \mathbf{u}}{\partial t}=-\mathcal{L}(\mathbf{u})-i f \mathbf{u}+\frac{\partial}{\partial z}\left(K_{u} \frac{\partial \mathbf{u}}{\partial z}\right)+\frac{d}{d \mathbf{x}}\left(K_{\mathbf{x}} \frac{d \mathbf{u}}{d \mathbf{x}}\right)+\frac{1}{\rho_{0}} \frac{d P_{i}}{d \mathbf{x}}-g \frac{d \zeta}{d \mathbf{x}}-\frac{1}{\rho_{0}} \frac{d P_{a}}{d \mathbf{x}}
$$

with the operator $\mathcal{L}=\left(u \frac{\partial}{\partial x}+v \frac{\partial}{\partial y}+w \frac{\partial}{\partial z}\right)$ and the complex derivative: $\frac{d}{d \mathbf{x}}=\frac{\partial}{\partial x}+i \frac{\partial}{\partial y} \cdot \mathcal{L}(\mathbf{u})$ represents the non-linear advection terms, $g$ the acceleration due to gravity, $\rho_{0}=1027.34 \mathrm{~kg} \mathrm{~m}^{-3}$ the reference water density, $f$ the Coriolis parameter, $K_{u}$ the vertical eddy viscosity and $K_{\mathbf{x}}$ the horizontal ones, $\zeta$ the sea surface height, and $P_{a}$ and $P_{i}$ the surface atmospheric and internal pressures, respectively. Since the model assumes the ocean to be hydrostatic, $P_{i}$ is derived from $\frac{\partial p}{\partial z}=\rho_{0} b$ with the buoyancy given by: $b=-g\left(\rho-\rho_{0}\right) / \rho_{0}$ and $\rho$ the water density. The vertical velocity follows from continuity. The surface boundary conditions are given by the zonal and meridional wind stress $\tau_{\mathbf{a}}$ computed from the 10-m wind $\left(\mathbf{u}_{10}\right)$ forcing using:

$$
\tau_{\mathbf{a}}=\rho_{a} C_{D} \mathbf{u}_{\mathbf{1 0}}^{2}
$$

where $C_{D}$ is the drag coefficient based on Charnock (1955) and $\rho_{a}$ the surface air density.

The evolution of a tracer $C$ is given by the advection-diffusion equation:

$$
\frac{\partial C}{\partial t}=-\left(\frac{\partial}{\partial x}[u C]+\frac{\partial}{\partial y}[v C]+\frac{\partial}{\partial z}[w C]\right)+\frac{\partial}{\partial z}\left(K_{C} \frac{\partial C}{\partial z}\right)+F
$$

where $K_{C}$ is the vertical diffusivity (the horizontal ones are set to 0 ). The source term $F$ consists of the solar irradiance and long-wave radiation for temperature. The salinity evolves with water fluxes induced by precipitation minus evaporation as well as river outflows. The surface boundary condition for temperature diffusion is the latent and sensible turbulent fluxes.

The eddy viscosity $\left(K_{u}=c_{\mu} \frac{k^{2}}{\varepsilon}\right)$ and vertical diffusivity for temperature $\left(K_{C}=c_{\mu}^{\prime} \frac{k^{2}}{\varepsilon}\right)$ are obtained from the two-equation $k-\varepsilon$ model (Burchard, 1998; Burchard and Bolding, 2001) which were chosen in this study for turbulence closure. The Canuto et al. (2001) version A (CA) stability functions $c_{\mu}$ and $c_{\mu}^{\prime}$ based on the non-dimensional shear and buoyancy numbers as described in Umlauf and Burchard (2005) are used to damp (enhance) turbulence in stably (unstably) stratified conditions. The turbulent kinetic energy (TKE, $k$ ) is modeled as:

$$
\frac{\partial k}{\partial t}=-\mathcal{L}(k)+\frac{\partial}{\partial z}\left(\nu_{k} \frac{\partial k}{\partial z}\right)+P+D-\varepsilon
$$


where the shear $P$ and the buoyancy $D$ contributions are functions of the vertical shear and the BruntVäisäla frequency, respectively. In the configuration used here, the advection term $\mathcal{L}(k)$ is neglected. The equation of the TKE dissipation rate $(\varepsilon)$ is given by:

$$
\frac{\partial \varepsilon}{\partial t}=\frac{\varepsilon}{k}\left(c_{\varepsilon 1} P+c_{\varepsilon 3} D-c_{\varepsilon 2} \varepsilon\right)+\frac{\partial}{\partial z}\left(\nu_{\varepsilon} \frac{\partial \varepsilon}{\partial z}\right)
$$

The empirical constants $c_{\varepsilon i}(=1.44,1.92,-0.63$ or 1) are taken from Burchard and Bolding (2001) and Umlauf (2003). A positive (negative) $c_{\varepsilon 3}$ is taken for unstable (stable) stratified flows (Canuto et al., 2001). The eddy viscosities $\nu_{k}$ and $\nu_{\varepsilon}$ are computed through constant Schmidt numbers as: $\nu_{k, \varepsilon}=\frac{K_{u}}{\sigma_{k, \varepsilon}}$ with $\sigma_{k}=1$ and $\sigma_{\varepsilon}=1.3$.

A constant flux condition is used at the sea surface for Eq. (4):

$$
\frac{\partial k}{\partial z}=0
$$

For Eq. (5), the Neuman-type surface boundary condition is:

$$
\frac{\partial \varepsilon}{\partial z}=\left(c_{\mu}^{0}\right)^{\frac{3}{4}} \frac{k^{\frac{3}{2}}}{\kappa\left(z_{0}+z^{\prime}\right)^{2}}
$$

where $c_{\mu}^{0}=0.077(\mathrm{CA}), z_{0}$ is the surface roughness length, $z^{\prime}$ the distance to the surface boundary, and $\kappa=0.4$ the von Karman constant.

\subsection{Coupled Wave-Ocean Model Framework}

The MARS3D has been coupled with the $3^{\text {rd }}$ generation wave model WAVEWATCHIII (WW3, Tolman (2014)), through OASIS-MCT (Voldoire et al., 2017). As listed on the diagram Figure 2, MARS3D sends 3 fields to WW3 and receives 17 fields from WW3. The framework was developed in a way to minimize the parameterizations of wave dependent variables within the ocean model. Thus coupling as many fields directly computed from the source function of WW3 and the model wave spectra as possible rather than relying on say wave height dependent parameterizations. The coupling between WW3 and MARS3D occurs every $100 \mathrm{~s}$. The coupling module performs distance weighted nearest neighbour interpolations from one regular model grid to the other based on 4 points using the Los Alamos National Laboratory SCRIP1.4 library (Jones, 1999).

The impact of waves on the ocean is modeled following the quasi-Eulerien framework (Ardhuin et al., 2008; Bennis et al., 2011) based on wave-averaged primitive equation limited to second order wave theory. In the coupled ocean-wave configuration Eq. (1) becomes:

$$
\begin{aligned}
\frac{\partial \hat{\mathbf{u}}}{\partial t}= & -\hat{\mathcal{L}}(\hat{\mathbf{u}})-i f \hat{\mathbf{u}}+\frac{\partial}{\partial z}\left(K_{u} \frac{\partial \hat{\mathbf{u}}}{\partial z}\right)+\frac{d}{d \mathbf{x}}\left(K_{\mathbf{x}} \frac{d \hat{\mathbf{u}}}{d \mathbf{x}}\right)+\frac{1}{\rho_{0}} \frac{d P_{i}}{d \mathbf{x}}-g \frac{d \zeta}{d \mathbf{x}}-\frac{1}{\rho_{0}} \frac{d P_{a}}{d \mathbf{x}} \\
& -i f \mathbf{u}_{\mathbf{s}}-i\left(\frac{\partial \hat{v}}{\partial x}-\frac{\partial \hat{u}}{\partial y}\right) \mathbf{u}_{\mathbf{s}}-\frac{\partial \hat{\mathbf{u}}}{\partial z} w_{s}-\frac{\partial J}{\partial \mathbf{x}}
\end{aligned}
$$


where $\hat{\mathbf{u}}$ is the quasi-Eulerien velocity defined as the difference between the Lagrangian velocity $\left(\mathbf{u}_{\mathbf{L}}\right)$ and the horizontal Stokes drift $\left(\mathbf{u}_{\mathbf{s}}\right)$ :

$$
\hat{\mathbf{u}}=\mathbf{u}_{\mathbf{L}}-\mathbf{u}_{\mathbf{s}}
$$

Both $\hat{\mathbf{u}}$ and $\mathbf{u}_{\mathbf{s}}$ satisfy continuity. In Eq. (8) $\hat{\mathcal{L}}=\left(\hat{u} \frac{\partial}{\partial x}+\hat{v} \frac{\partial}{\partial y}+\hat{w} \frac{\partial}{\partial z}\right)$ and $w_{s}$ is the vertical component of the Stokes drift. New wave dependent terms appear on the right hand side terms of Eq. (8): the Stokes-Coriolis force $\left(i f \mathbf{u}_{\mathbf{s}}\right)$, the Stokes vortex force $\left(-i\left(\frac{\partial \hat{u}}{\partial x}-\frac{\partial \hat{u}}{\partial y}\right) \mathbf{u}_{\mathbf{s}}-\frac{\partial \hat{\mathbf{u}}}{\partial z} w_{s}\right)$, and the wave-induced Bernoulli head pressure (J, Smith (2006)). In this configuration, the model remains hydrostatic and the impact of the Stokes shear on the pressure gradient (see Suzuki and Fox-Kemper, 2016) is not taken into account. Note that only surface Stokes drift fields are sent by the wave model to the ocean model along with the Stokes transport. These are then used to approximate vertical Stokes profiles based on Breivik et al. (2014).

In addition to these modifications in current equations, the boundary conditions are changed. The surface stress felt by the ocean is no longer $\tau_{\mathbf{a}}$ but:

$$
\tau_{\text {surf }}=\tau_{\mathbf{a}}-\tau_{\mathbf{a w}}+\tau_{\mathbf{w o}}
$$

where $\tau_{\text {aw }}$ corresponds to the amount of momentum used by wave formation and $\tau_{\mathbf{w o}}$ to the breaking wave induced stress transferred to the ocean. These fields, provided by WW3, are derived from the input and dissipation source functions (Tolman, 2014). At the bottom boundary, the stress due to the waves is added to the bottom friction. It is computed from the bottom orbital velocities $\mathbf{u}_{\mathbf{b w}}$ and a wave friction factor following Soulsby et al. (1993). For the wave friction factor the parameterisation of Soulsby (1997) (their Eq. 62a) is used.

Waves impact the advection of tracers which is done by the Lagrangian velocity. Thus, with waves Eq. (3) becomes:

$$
\frac{\partial C}{\partial t}=-\left(\frac{\partial}{\partial x}\left[\left(\hat{u}+u_{s}\right) C\right]+\frac{\partial}{\partial y}\left[\left(\hat{v}+v_{s}\right) C\right]+\frac{\partial}{\partial z}\left[\left(\hat{w}+w_{s}\right) C\right]\right)+\frac{\partial}{\partial z}\left(K_{C} \frac{\partial C}{\partial z}\right)
$$

Waves effects have also to be considered as impacting eddy viscosity and diffusion terms in Eqs. (8) and (11). This is achieved by modifying the surface boundary conditions of the turbulent closure equations and adding a new source terms within the bottom boundary layer. The TKE production term added to the right hand sides of Eqs. (4) and (5) is taken from Walstra et al. (2000). For $k$, it stands as:

$$
P_{w}^{b o t}(z)=\frac{2 \Phi_{b b l}}{\delta}\left(1-\frac{d+\zeta-z}{\delta}\right) \quad \text { for } d+\zeta-z \leq \delta
$$

where $\Phi_{b b l}$ is the wave energy dissipation due to bottom friction sent by WW3 to MARS3D and $d$ the topography. The bottom wave boundary layer thickness $\delta$ is derived following Grant and Madsen (1982) 
(their Eqs. 5 and 6) based on the wave friction factor. These depend on the bottom orbital velocities $\mathbf{u}_{\mathrm{bw}}$ and the mean wave period $T_{m 1}$ provided by WW3.

Thus Eqs. (4) and (5) become:

$$
\begin{array}{r}
\frac{\partial k}{\partial t}=-\mathcal{L}(k)+\frac{\partial}{\partial z}\left(\nu_{k} \frac{\partial k}{\partial z}\right)+P+D-\varepsilon \\
+P_{w}^{b o t} \\
\frac{\partial \varepsilon}{\partial t}=\frac{\varepsilon}{k}\left(c_{\varepsilon 1} P+c_{\varepsilon 3} D-c_{\varepsilon 2} \varepsilon\right)+\frac{\partial}{\partial z}\left(\nu_{\varepsilon} \frac{\partial \varepsilon}{\partial z}\right) \\
+\frac{\varepsilon}{k} c_{\varepsilon 1} P_{w}^{b o t}
\end{array}
$$

For these two equations, the Neuman-type boundary conditions taking the waves into account at the sea surface are given by:

$$
\frac{\partial k}{\partial z}=\Phi_{o c}
$$

$$
\frac{\partial \varepsilon}{\partial z}=\left(c_{\mu}^{0}\right)^{\frac{3}{4}}\left[\frac{k^{\frac{3}{2}}}{\kappa\left(z^{\prime}+z_{0}\right)^{2}}+\left(c_{\mu}^{0}\right)^{\frac{3}{4}} \frac{\frac{3}{2} \sigma_{k} \Phi_{o c}}{c_{\mu} \kappa^{2}\left(z^{\prime}+z_{0}\right)^{2}}\right]
$$

with $c_{\mu}=0.09$ (Burchard, 2001) and $\Phi_{o c}$ the wave to ocean energy flux provided by WW3 accounting for the injection of TKE from breaking waves. Similarly to $\tau_{w o}, \Phi_{o c}$ is derived from the parameterisation chosen for the dissipation source function in WW3. Note that $\Phi_{o c}$ replaces $c_{w} u_{*}^{3}$ from the Craig and Banner (1994) model for the wave enhanced layer in the boundary conditions reported in Burchard (2002). Rather than considering the surface wave TKE contribution as a flux, it could be inputted as a vertically distributed (at the scale of the heights of breaking waves) production term similar to $P_{w}^{b o t}$. Given the very high mixing there is very little difference between these approaches Rascle et al. (2013).

\subsection{Case Study}

A realistic case study was devised to evaluate the impact of waves on the Ushant tidal front under low to moderate winds. Given the relatively short period over which consistent wind and wave conditions prevail, the simulations were run only for a couple of days. This does not allow for a full tidal decomposition of the results. However, choosing a period in which waves propagate consistently in one direction allows straight forward contrasting of different wave-tide alignments. Models (MARS3D, WW3) were set-up to best represent the conditions measured during the FROMVAR (Front de Marée Variable) field campaign between the 02 and 04 September 2011. An anticyclonic circulation was present at the beginning of the campaign with very weak winds and calm seas. With the arrival of an atmospheric front around 1700 UTC on 02 September, southwesterly winds gradually intensified to reach a local maximum of $14 \mathrm{~m} \mathrm{~s}^{-1}$ on 
03 September around 1600 UTC. The coupled simulations were started on 02 September at 0600 UTC but in the following analysis, model outputs are only considered starting at 1200 UTC, allowing for adequate wave spin up from calm conditions.

\subsection{Model Configurations}

The models were run at high horizontal resolution (500 $\mathrm{m}$ for MARS3D and $1500 \mathrm{~m}$ for WW3) over a domain spanning about 160 by $160 \mathrm{~km}^{2}$ (Fig. 1) with hourly outputs. The model configurations chosen are those used for operational purposes as part of the "Modelling and Analysis for Coastal Research" (MARC) project (https://marc.ifremer.fr). A stand-alone MARS3D run (version V11.0_OAW01) was spun up starting January 2011 using a Bay of Biscay configuration with a horizontal resolution of $2.5 \mathrm{~km}$ similar to that used operationally for Prévimer (Lazure et al., 2009) (precursor to the MARC project). A fine resolution zoom $(500 \mathrm{~m})$ over the Iroise Sea was embedded into the regional grid and integrated through 2-way AGRIF nesting (Debreu et al., 2008). This zoom provided the initial and lateral boundary conditions for the MARS3D simulations used in the present study. Run from 0600 UTC on 02 September 2011 to 0000 UTC on 04 September 2011, the stand-alone MARS3D simulation analysed herein gives almost identical outputs to the zoom. The chosen model configuration and forcing were shown to best reproduce the front. Choice of the turbulence scheme was motivated by previous model development (Bennis et al., 2011).

MARS3D was forced with tidal components taken from the global Finite Element Solution tide model FES2004 (Lyard et al., 2006) and atmospheric forcing fields consistent with those used to force the Bay of Biscay run. These atmospheric forcing fields come from the operational French forecast model AROME with a temporal resolution of 1 hour and a spatial resolution of $0.025^{\circ}(\sim 2.5 \mathrm{~km})$. AROME provides the following variables to MARS3D: 2-meter air temperature, atmospheric pressure, relative humidity, 10-m zonal and meridional wind components, and cloud cover. Heat fluxes are internally computed based on the NOMADS-2 parameterisation for solar fluxes proposed by Gill (1982), the "EDF" parameterisation for thermal fluxes described in Dumas and Langlois (2009), and COARE 3.0 (Fairall et al., 2003) for the turbulent fluxes. MARS3D operates on an Arakawa $\mathrm{C}$ grid with topographically conformal vertical coordinate $(\sigma)$ system. The vertical resolution was set to $40 \sigma$ levels. Figure 3 shows the mean surface $(0-30 \mathrm{~m})$ vertical resolution over a tidal cycle. The top sigma level is about $15 \mathrm{~cm}$ below the sea surface. Each subsequent sigma level is about $30 \mathrm{~cm}$ deep over the top $2.4 \mathrm{~m}$ of the water column, then gradually increase in thickness to be around $0.5 \mathrm{~m}$ at $5 \mathrm{~m}$ below the water line. Surface diagnostics of the current and temperature equations shown in the results section are from the top sigma level while near surface 
$k-\varepsilon$ diagnostics are from the top mid-level. The model automatically controls the timestep starting from a maximum timestep of $20 \mathrm{~s}$ and a critical Courant-Friedrichs-Lewy (CFL) condition set to 0.6. The QUICK (Kowalik and Murty, 1993) numerical scheme is used for the advection of currents. For tracer (temperature and salinity) a fifth order upwind scheme is used for horizontal advection and an upwind compact and conservative scheme is used for vertical advection (Debreu and Duhaut, 2011). These are employed in a three dimensional two-step MACHO method (Leonard et al., 1996). An implicit scheme is used for vertical diffusion of currents and tracers. The forcing, parameterisations, and numerical schemes chosen correspond to those used in the bay of Biscay configuration which combined with the AGRIF downscaling method allow for the best representation of the Ushant front.

In WW3 (adapted from v5.16), the spectral space was discretized using 32 frequencies ranging from 0.0373 to $0.7159 \mathrm{~Hz}$ in $10 \%$ steps $\left(f_{i+1}=1.1 f_{i}\right.$, where $i$ is a discrete grid counter and $f$ the frequency) with 24 directions $\left(\Delta \theta=15^{\circ}\right)$. The overall timestep was set to $100 \mathrm{~s}$, the spatial (CFL without currents) and spectral (refraction) propagation timesteps to $25 \mathrm{~s}$, and the integration of the source function to $10 \mathrm{~s}$. The physics package used is ST3 which prescribes an $f^{-5}$ spectral tail outside the model frequency range (Bidlot et al., 2005; Tolman et al., 2014). This set of source function parameterizations was chosen for their ability to reproduce the variability in the drag coefficient. For a review of the performances of the different physics packages available see Stopa et al. (2016). The model was started from calm conditions $\left(H_{s}=0\right)$ and forced at the boundaries by 3 hourly energy spectra produced by the HOMERE reanalysis (Boudière et al., 2013) at the open boundaries. The atmospheric forcing for WW3 (zonal and meridional components of the $10 \mathrm{~m}$ wind) are taken from the AROME fields used to force MARS3D.

\section{Results}

The wave impact onto the ocean is analysed by contrasting results of the coupled MARS3D-WW3 run with those of the stand-alone MARS3D run. The following paragraphs focus on the northern extension of the front around a longitudinal transect at $48.8^{\circ} \mathrm{N}$ as well as the two nearly homogeneous areas Z1 and Z2 located on each side of the Ushant front (see Fig. 1) where the water depth is $116.2 \pm 0.97 \mathrm{~m}$ and 98 $\pm 2.2 \mathrm{~m}$, respectively.

\subsection{Environmental Conditions Simulated}

Figure 4 shows time series of the key variables describing the environmental conditions over the simulated period. Average conditions are plotted for the whole domain (black curves) as well as for two 
equal sized zones, one on either side of the front: Z1 (stratified, red curve) and Z2 (well mixed, blue curve); c.f. Figure 1. As shown below, the tidal signal of the current (rather than the sea level) is central to understand the impact of waves on the front. Throughout the paper, particular attention will be drawn to the relative alignment between current and wave propagation directions. Therefore, periods when waves propagate with (against) the tide roughly corresponding to flood (ebb) or northeastwards (southwestwards) flow are highlighted with coral (blue) shadings on the figures.

The mean winds grow gradually from $\sim 3$ to $\sim 8 \mathrm{~m} \mathrm{~s}^{-1}$ between 1200 UTC on 02 September and 1800 UTC on 03 September before decreasing shortly until 2100 UTC and increasing again up to $\sim 9 \mathrm{~m} \mathrm{~s}^{-1}$ at the end of the simulation (Fig. 4c). A similar pattern is observed in Z1 and Z2, though winds reach a maximum on 03 September of $9.6 \mathrm{~m} \mathrm{~s}^{-1}$ at 1300 UTC in Z1 and of $8.4 \mathrm{~m} \mathrm{~s}^{-1}$ at 1800 UTC in Z2. Also, unlike in Z1 or when averaged over the whole domain, winds in Z2 do not increase again after 2100 UTC on 03 September. They blow to the north-northeast throughout the majority of the simulation but for the first and last few hours when average directions display more variability (Fig. 4d).

The tidal cycle as described by the sea level (Fig. 4a), current intensity (Fig. 4e), and direction (Fig. 4f) is semi-diurnal. Three tidal cycles with a tidal range of about $6 \mathrm{~m}$ are resolved over the study period starting at low water. The magnitude of the tidal current (Fig. 4e) varies between $\sim 0.4$ and $\sim 0.8 \mathrm{~m}$ $\mathrm{s}^{-1}$ on average over the whole domain and Z1. This range is intensified near the coast as shown by the temporal averages in $\mathrm{Z} 2$ that vary between $\sim 0.2$ and $\sim 1.2 \mathrm{~m} \mathrm{~s}^{-1}$. Currents are the strongest around times of high and low water as is the case for progressive tidal waves.

From 2000 UTC on 02 September, the significant wave height increases from $<1 \mathrm{~m}$ to $\sim 2 \mathrm{~m}$ (Fig. 4b) and its temporal evolution follows that of the wind (Fig. 4c). The main spectral wave peak corresponds to a swell system (see wave periods in Fig. 4b). Waves propagate to the northeast (Fig. 4h) and are roughly aligned with the wind (Fig. 4d). The intensity of the Stokes drift (Fig. 4g) increases as the wind and the waves grow. The wave period is seen to decrease smoothly over time. It does not appear to be influenced by the tide. However, the Stokes drift presents two maxima $0.053-0.076 \mathrm{~m} \mathrm{~s}^{-1}$ and $0.073-0.1 \mathrm{~m} \mathrm{~s}^{-1}$ around 0200 and 1300 UTC on 03 September, respectively. The Stokes drift appears to be intensified during ebb flow when waves roughly oppose the tidal current (blue shaded periods). The significant wave height also displays local maxima around these times. A decrease in the Stokes drift and in the significant wave height is observed during the two last flood tides of the study period. This aligns with the concept that a wave-opposing current steepens (decrease in mean wavelength, increase in amplitude). A wave-opposing current compresses the wave field while a wave-following current stretches, thus flattens it (Ardhuin et al., 2017; Phillips, 1984). As the Stokes drift is proportional to the square of the steepness of the waves it 
increases.

\subsection{Frontal Displacement and the Temperature Equation}

The sea surface temperature (SST) front is well represented in both MARS3D runs. It is characterized by a strong SST gradient between $4.9^{\circ} \mathrm{W}$ and $5.1^{\circ} \mathrm{W}$ with warm $\left(\mathrm{SST}>15.5^{\circ} \mathrm{C}\right)$ stratified waters offshore and cold (SST $<14{ }^{\circ} \mathrm{C}$ ) well mixed waters close to the coast (Fig. 1a). The mixed layer whose depth is computed based on a temperature criterion of $0.2^{\circ} \mathrm{C}$ absolute difference from surface, is around $10-20 \mathrm{~m}$ deep on the stratified side and reaches the sea floor on the mixed side (Fig. 1b). Note that computing the mixed layer depth based on a density criterion does not significantly alter the results. Looking at longitude-time Hovmöller diagrams of the SST along the transect at $48.8^{\circ} \mathrm{N}$ clearly shows the zonal displacement of the Ushant front with the tidal flow (Fig. 5b). As expected, it is seen to advance towards the shore with the flood and retreat offshore with the ebb flow in both the stand-alone and coupled simulations as illustrated in Figure 5b. The zonal displacement at $48.8^{\circ} \mathrm{N}$ is not in phase with the mean sea level (MSL, Fig. 5a) but rather with the zonal surface current (Fig. 5d) showing how tidal advection drives the front's position.

A surface negative temperature anomaly of up to $\sim 1.5^{\circ} \mathrm{C}$ in the stratified side (Fig. 5c) is visible in the coupled run compared to the stand-alone run. This cooling is pronounced $\left(>1^{\circ} \mathrm{C}\right)$ within 2 to $4 \mathrm{~km}$ west of the front location in the stand-alone MARS3D run between 0100 and 1800 UTC on 03 September but extends over 10 kilometers further. The differences in temperature and displacement intensify during the simulated period as the waves grow. No clear link is observed between the intensity of the SST response and the tidal cycle. A wave induced cooling can be expected due to wave surface breaking induced mixing (Eqs. 15, 16) which would only have visible impacts on the temperature on the stratified part. However, the analysis of the location of the maximum SST gradient along $48.8^{\circ} \mathrm{N}$ reveals that this cooling is associated with a westward displacement of the Ushant front. Differences in the surface tracer advecting velocities between the coupled and stand-alone runs are mostly negative around the position of the front (Fig. 5e). This indicates a reduction of the eastward advection and an increase of the westward advection. On the mixed side of the front, the differences are positive indicating an increased advection to the east in line with the Stokes' drift and regardless of tidal phase. On the stratified side of the front, changes in tracer advection are more variable with both positive and negative regions and periods without a clear relation to the tidal phase. The mixed layer depth is seen to reach the sea bottom within a few kilometers west of the SST front as detected in the uncoupled run further hinting at the westward displacement of the front due to waves (Fig. 5f). A deepening of the mixed layer of 5-10 $\mathrm{m}$ is also seen 
throughout the stratified side outside of the frontal region. It intensifies over time and with proximity to the front.

The location of the SST front is obtained based on the maximum values of the SST gradient or mixed layer depth (MLD) obtained by a Sobel filter (Pringle, 1969). Its temporal evolution at $48.8^{\circ} \mathrm{N}$ is shown in Figure 6a. Both types of estimation are consistent between them. The one based on the MLD gives a more regular forehead position that clearly follows the tidal flows. The SST based one appears noisier. When wave-current interactions (coupled run) are modeled, the position deduced from the SST gradient is up to $4 \mathrm{~km}$ further west than in the stand-alone run (Fig. 6b). Tracking a specific isotherm such as $14.7^{\circ} \mathrm{C}$ gives comparable results (not shown). The offset of the position deduced from the maximum gradient of MLD deduced from the coupled and stand-alone runs reaches $3.5 \mathrm{~km}$ at the end of the simulation, tracking the SST offset (Fig. 6b). Although results are shown for a specific latitude, the impact of waves is clearly visible all along the Ushant front. As illustrated in Figure 7, the front has similar meanders in the stand-alone (panel a) and coupled (panel b) runs. Mapping the difference in SST between the coupled and the stand-alone run (Fig. 7c) clearly reveals cooling on the stratified side of the front. Some positive differences in SST are visible in the southern extension of the front where meanders are stronger, particularly near the Ushant Island. Presence of the island and shallow bathymetry there complicates the dynamics and wave interactions.

Positive salinity and density anomalies accompany the negative surface temperature anomaly on the west side of the front (Fig. 8). The anomalies extend to the MLD on the stratified side of the front without any clear variation of magnitude with depth. Up to $\sim 5 \mathrm{~m}$ beneath the MLD of the stand-alone run, the anomalies are of opposite sign as can be expected from enhanced mixing. This is accompanied with a deepening of the MLD of $\sim 2-3 \mathrm{~m}$. No significant differences in temperature and salinity, hence neither in density, are found at depth between the run with or without waves (a full depth version of Figure 8 can be found in Supplementary Materials, c.f. S1). This suggests that only the surface front is impacted by waves under the forcing considered here. The waves impact on the tracer fields is however clearly seen several meters below the mixed layer.

Three physical processes could explain the SST cooling and the westward shift of the SST front in the coupled MARS3D-WW3 compared to the stand-alone MARS3D run:

1. horizontal advection of SST gradient to the west replacing warm stratified waters by cold waters from the mixed side of the front;

2. increase of the vertical mixing through additional injection of TKE in ocean due to the presence of waves; 
3. changes in vertical entrainment at the MLD bottom bringing cold water up due to wave induced changes in mean vertical velocities.

Changes of vertical entrainment as computed from the vertical velocity using the continuity equation are not significant. The leading order processes driving the temperature evolution are advection and vertical diffusion. Contrasting the budget of the temperature equation of the coupled and stand-alone runs (Eqs. 3 and 11, respectively) allows to determine which of these two processes changes more in response to wave coupling. Figure 9 shows Hovmöller plots at $48.8^{\circ} \mathrm{N}$ of the different terms of the temperature equation for the stand-alone MARS3D run (panels a and b) and the difference between the coupled wave-ocean and the stand-alone MARS3D runs (panels c to e). The temporal trend term of the stand-alone run is not shown because it is very closed to the total advection term as expected in a tidally dominated environment. Note also that budget terms are hourly averaged rather than instantaneous as in previous figures. The semi diurnal tidal signal is clearly visible in the total advection (Fig. 9a) but not in the vertical diffusion term (Fig. 9b) which is negative on the well mixed side of the front and positive on the stratified side. The plots of differences reveal that the changes in the advective terms (Fig. 9d) are larger around the front than the changes in the vertical diffusion (Fig. 9e). Indeed, the difference in the temperature trend (Fig. 9c) follows that of the total advection revealing cooling near the front, particularly during flood tide. Figure 9e does however clearly show an increase in vertical diffusion on the warm side of the front at times.

\subsection{Current and $k-\varepsilon$ Equations}

To further explore the dynamics and energetics responsible for the wave induced changes described above, current and $k-\varepsilon$ equation budgets computed from the coupled and stand-alone runs are compared. Figures 10a,b,d, and e show vertical profiles of the horizontal components of the Eulerian and quasiEulerian velocities averaged in Z1 (red) and Z2 (blue) for two instants: one when waves and currents are opposed (1200 UTC on 03 September, top row) and one when they follow each other (1800 UTC on 03 September, bottom row). The general behaviour on both the stratified (Z1) and mixed (Z2) sides of the front is similar. Currents opposing (following) to wave propagation are found to increase (decrease). The vertical profiles all clearly feature a near surface gradient, although the mean flow is driven largely by barotropic tides. The impact of waves on currents is clearly enhanced near surface but reaches below the MLD which is around $15.3 \mathrm{~m}$ and $15.8 \mathrm{~m}$ at $1200 \mathrm{UTC}$ and $13.8 \mathrm{~m}$ and $14.1 \mathrm{~m}$ at $1800 \mathrm{UTC}$ in Z1 in the stand-alone and coupled run, respectively. The vertical eddy viscosities (Fig. 10c and f) are seen to increase within the top $\sim 10-15 \mathrm{~m}$ of the water column in both cases on either side of the front. The 
increased mixing leading to reduced vertical gradients can explain the increased (decreased) quasi-Eulerian velocities in condition of wave-opposing (-following) currents. A positive vertical gradient (shear), likely due to Ekman dynamics, exists at all times in both the zonal and the meridional velocity components. The resulting down-gradient turbulent diffusion leads to an increase in magnitude of the upper ocean velocity when negative and a decrease in the magnitude when positive.

The impact of waves on the magnitude of the near-surface tidal current is further analysed through time series of differences between the magnitude of the quasi-Eulerian current in the coupled run and that of the Eulerian current in the stand-alone run (Fig. 11a). The differences of the current magnitudes averaged over Z1 reach $0.080 \pm 0.007 \mathrm{~m} \mathrm{~s}^{-1}$ during the ebb flow on 03 September at 1200 UTC and $0.073 \pm 0.010 \mathrm{~m} \mathrm{~s}^{-1}$ during the rising tide at 1800 UTC on 03 September. This equates to changes in the (quasi-)Eulerian surface current magnitude of up to $\pm 17.5 \%$ relative to the Eulerian current magnitude of the stand-alone run in Z1. Changes on the mixed side of the front (Z2) are relatively smaller, amounting to $+11 \%$ and $-16.7 \%$. The differences in the zonal (meridional) (quasi-)Eulerian current components (see Supplementary Material S2 and S3) are for the most part negative indicating that east(north)ward flows are reduced and west(south)ward flows are enhanced. Note that the magnitude of the differences in (quasi-)Eulerian currents is for the most part smaller than the magnitude of the Stokes drift (see Fig. 4g). Indeed, comparing the Lagrangian velocity of the coupled run to the Eulerian velocity of the stand-alone run suggests the Stokes drift tends to compensate the changes in (quasi-)Eulerian currents. It increases (decreases) the wave-following (-opposing) currents by $<3 \%$ outside of the frontal region, accounting for the positive $\Delta\left|\mathbf{u}_{\mathbf{L}}\right|$ on the well mixed side in Figure 5e. However, as shown in Figure 5e, this is not the case in close proximity of the temperature front where the Lagrangian velocity in the coupled run is smaller than the Eulerian velocity of the stand-alone run during flood tides and larger during ebb flow.

The analysis of the current equation budget allows to evaluate the main physical balances at play. Given the budget of zonal and meridional currents, it is easy to derive that of the current magnitude. The evolution of the horizontal current follows from Eqs. (1) and (8):

$$
\frac{\partial \sqrt{u^{2}+v^{2}}}{\partial t}=\frac{1}{\sqrt{u^{2}+v^{2}}}\left(u \frac{\partial u}{\partial t}+v \frac{\partial v}{\partial t}\right)
$$

Note that doing so eliminates the (quasi-)Eulerian Coriolis terms, meaning that the temporal evolution of the current magnitude follows the pressure gradient term at first order (Fig. 11b). Plotting the terms of the equations for the zonal and meridional components (see Supplementary Material S2 and S3) rather than the module clearly shows that the main evolution of the currents is driven from a balance between the pressure gradient and the Coriolis force, i.e. tides. Figure 11b shows the time series of the domain 
averaged terms contributing to the evolution of current magnitude. Starting around 2000 UTC on 02 September a separation is visible between the terms of the stand-alone (solid curves) and the coupled (dashed curves) MARS3D outputs in both the temporal trend (black curves), the pressure terms (purple curves), and the vertical diffusion term (light blue curves). Note that the addition of the wave induced pressure $(J)$ does not significantly alter the pressure gradient terms. The observed offset between the solid and dashed purple curves reflects differences between the Eulerian current of the stand-alone run and the quasi-Eulerian current of the coupled run. When looking at the zonal and meridional components (Supplementary Material S2 and S3), the change in (quasi-)Eulerian currents is reflected in the (quasi)Eulerian Coriolis terms (if $\mathbf{u}$ ). Time series of the difference between the coupled and the stand-alone MARS3D fields (Fig. 11c) clearly show that as the waves start to grow the Stokes-Coriolis term grows. The difference in trend of the quasi-Eulerian velocity in the coupled run and the Eulerian velocity in the stand-alone run arises from changes in the vertical diffusion term and the Coriolis terms which evolve in opposite phase. The balance described above was verified to hold in average not only over the whole domain, but also in Z1 and Z2.

Changes in the vertical diffusion term arise from changes in the surface stress, the vertical shear, and the eddy viscosity (see Eqs. 1 and 8). The surface stress acting upon the ocean (Eq. 10 vs. Eq. 2) is reduced when waves and currents are aligned, but enhanced when opposed, with changes of the order of $\pm 10 \%$ relative to $\tau_{a}$. The near surface shear is reduced regardless of wave-current alignment. The near surface eddy viscosity is enhanced on average by a factor of $\mathcal{O}(10)$ in the coupled run compared to the stand-alone run due to changes in the $k-\varepsilon$ equations (Eqs. 12-16 vs. Eqs. 4-7). As discussed in Section 2.2 , the surface boundary conditions are modified when wave effects as derived from WW3 model are taken in account. Consequently, the near surface TKE (Fig. 12a) and the dissipation $\varepsilon$ (not shown) are seen to increase as the waves grow displaying two maxima similar to the Stokes drift magnitude and $H_{s}$.

As variations of the TKE dissipation rate largely reflect those of TKE, more insights may be gained by looking at the evolution of the dissipation length scale $L$ derived via the Taylor (1935) scaling:

$$
L=C_{L} \frac{k^{3 / 2}}{\varepsilon}
$$

where the proportionality constant $C_{L}=\left(c_{\mu}^{0}\right)^{\frac{3}{4}} \simeq 0.146$ is derived from the stability function $c_{\mu}$ for $D=$ 0 and $P=\varepsilon$ (see Burchard, 2002). While the near surface $L$ in the stand-alone run is nearly constant and equal to $0.24 \pm 0.031 \mathrm{~m}$, it increases from 0.23 to $0.48 \mathrm{~m}$ when averaged over the whole domain in the coupled run (Fig. 12b), mimicking the evolution of the $H_{s}$ and the Stokes drift. Note that $L$ in the stand-alone run is directly proportional to $z_{0}$ which was set to $0.4 \mathrm{~m}$. This is equivalent to conditions with $H_{s}$ equal to $0.67 \mathrm{~m}\left(z_{0}=0.6 H_{s}\right.$, following the scaling law proposed by Terray et al. (1996) with the 
proportionality coefficient of Soloviev and Lukas (2003)) which roughly corresponds to the wave heights at the beginning of the coupled simulation. Note that other scalings of $z_{0}$ as a function of $H_{s}$ can be found in the literature (Rascle et al., 2006), particularly $z_{0}=1.6 H_{s}$ (Terray et al., 2000) which unlike the scaling proposed by Soloviev and Lukas (2003) includes the contribution of swell. Taking $z_{0}=1.6 H_{s}$ increases mixing in the coupled run leading to an unrealistically flat temperature front at the end of the 2.5 days simulation. It does however have no substantial impact on the stand-alone run. As $z_{0}$ appear in the denominator of the surface boundary condition for $\varepsilon$ (Eq. 16), increasing it leads to a reduction in $\varepsilon$ and thus an increase in $k$ and $L$. This in turn further increases the diffusivities, leading to a further deepening of the mixed layer and cooling of the surface.

The leading order terms of the near surface TKE budget in both the stand-alone and coupled runs are the vertical diffusion and the TKE dissipation rate (Fig. 13b). These are substantially enhanced in the coupled run. The vertical diffusion term displays a clear tidal variation in the stand-alone run (not shown), varying with current magnitude, being minimum (maximum) an hour before the current maximum (minimum). The tidal signature in the vertical diffusion term is no longer visible in the coupled run. Rather, like the TKE dissipation rate, it increases as the waves growth and the two periods of enhancement are clearly seen in the domain mean time series. The shear production term is seen to be dampened by waves in the near surface $k$ equation (Fig. 13a) as expected from the increased mixing.

\subsection{Analysis of Partially Coupled Runs}

In order to better identify the different processes at play, a series of partially coupled model experiments were run. Four partially coupled runs are considered in this section: a run in which all Stokes drift related processes are included in the current and tracer equations (STOKESALLonly), a run where only the Stokes advection terms are included in the tracer equations (STOKESADVonly), a run where only the StokesCoriolis terms are included in the current equations (STOKESCORonly), and a run where waves are only taken into account in the turbulence closure model (WAVETURBonly). For the first three partially coupled runs, the turbulence equations and surface boundary conditions remain unchanged, i.e. there is no increase in $K_{u}$ or $K_{C}$ and the vertical diffusion terms are the same as in the stand-alone MARS3D run. In all the above mentioned runs the vertical boundary condition of the current equations is that of the stand-alone run, i.e. the impact of the waves on the surface stress is ignored. Partially coupled runs where only the stress was changed (not shown) revealed that it does not alter the front position significantly.

Figure $14 \mathrm{~b}$ shows the cross-front SST at $48.8^{\circ} \mathrm{N}$ for the partially coupled runs as well as the stand-alone 
and the fully coupled runs. The SST curves are centered on the position of the $14.7^{\circ} \mathrm{C}$ isotherm in the stand-alone run. This snapshot illustrates the relative frontal displacement between the runs once the wave impact is visible. As expected, including only the Stokes advection terms (STOKESADVonly) leads to an eastward shift of the front, in the direction of the wave propagation. Only including the Stokes-Coriolis terms (STOKESCORonly) can have the opposite effect displacing the front westwards as shown for the considered instant. The front remains however to the east of that in the fully coupled run. As mentioned in the previous section, the Stokes-Vortex force has minimal impact: the run STOKESALLonly shows the combined effect of the Stokes-Coriolis force on the current and of the Stokes advection on tracers. It leads to a slight eastward displacement of the front relative to the stand-alone position. The Stokes-Coriolis force can act to increase the eastward displacement along with the Stokes drift, depending on the relative alignment between the currents and wave propagation. These partially coupled experiments suggest that the wave induced mixing is largely responsible for the frontal displacement. Indeed, the frontal position of the WAVETURBonly run follows that of the fully coupled run very closely and is at times even further to the west than in the fully coupled run. Although results in Section 3.1 suggest that for temperature wave induced changes in mixing are less important than wave induced changes in advection, this is not the case for currents. Thus, it is the impact of wave mixing on currents rather than on temperature that is the key process explaining the main difference between stand-alone and coupled runs.

Vertical profiles of the (quasi-)Eulerian and Lagrangian velocities along the front (Fig. 14) clearly show strong baroclinicity on the stratified side of the front. Profiles of the zonal and meridional current components display a positive vertical gradient which is more pronounced on the stratified side of the front. Thus, when the flow is positive (negative)/north-eastwards (south-westwards) the flow is stronger (weaker) on the stratified side of the front than on the mixed side. Consequentially, once the front is displaced westwards due to mixing, the surface velocities within several kilometers of the front as identified by the $14.7^{\circ} \mathrm{C}$ isotherm are homogenised: their intensities have increased when negative and decreased when positive. This shift of regime induced by wave mixing within the frontal area has a greater impact than the changes related to the Stokes drift.

\section{Discussion}

The realistic model simulations described above reveal the role of gravity waves in modifying the position of a surface thermal tidal front. Waves propagating shore-ward roughly perpendicularly to the front result in a displacement of the front in the opposite direction of the wave propagation and Stokes drift. This is due to an increase (decrease) of the quasi-Eulerian currents when waves oppose (follow) 
them which is more important than the Stokes drift around the front. The following paragraphs draw parallels between these results and previous work before exposing avenues for future development and discussing broader implications.

\subsection{Comparison to Laboratory Experiments}

The simulated quasi-Eulerian current response to waves is in line with early laboratory measurements (Bakker and Van Doorn, 1978; Kemp and Simons, 1982, 1983; Klopman, 1994, 1993; Umeyama, 2005). Full depth Eulerian-mean current records show an increase (reduction) in surface currents when opposed (following) to waves compared to the classic logarithmic law of the wall current profiles without waves. These flume experiments were geared mainly towards analysing how waves alter currents in the bottom boundary for different bed roughness. Neertheless, they show systematic changes in the surface currents. They are regularly considered as the "truth" for wave-current interaction model developments although being based on paddle generated non-breaking wave trains which do not have the three dimensionality and spectral spread comparable to real wave fields. It had been argued that the observed wave impact was due to the presence of lateral boundaries which induce Langmuir circulations (Dingemans et al., 1996). However, transverse circulations due to sidewalls were shown to only weakly impact velocity profiles (Groeneweg and Battjes, 2003).

The mean velocity shear was also observed to be altered by waves in the aforementioned laboratory records: it increased when waves propagated against the current and decreased or even changed sign when waves and currents propagated in the same direction. Turbulent intensity measurements from Umeyama (2005) suggest that changes in shear were due to the wave-current Reynolds stresses which arise from the phase averaged correlation between the horizontal and vertical particle motion. The sign of the wavecurrent Reynolds stress always opposes that of wave propagation. Thus, for wave-following (-opposing) the current, it is of the opposite (same) sign to the current shear leading to a reduced (enhanced) shear. Wave-current Reynolds stresses are not directly included. As it stands, the coupled framework presented here leads to a reduction of the vertical velocity shear in the surface layer due to the increased wave mixing regardless of the relative alignment between currents and waves. Yet the expected enhancement of the quasi-Eulerian current is reproduced during wave-opposing periods. This is only because mixing homogenises the positive background surface current gradient which exists regardless of waves under the simulated wind conditions. 


\subsection{Comparison to Wave-Current Models and Boundary Layer Theories}

Several analytical 1D wave-current models and boundary layer theories have been proposed to reproduce the quasi-Eulerian current response observed in the laboratory experiments detailed above. Relying on either the surface distortion of the depth dependent eddy-viscosities (Huang and Mei, 2003), parameterization of wave induced second order stresses (You, 1996), or a mixing length hypothesis (Yang et al., 2006) they are capable of roughly replicating measured vertical velocity profiles. More recently, Olabarrieta et al. (2010) proposed a 3D non hydrostatic Eulerian wave-current model using a one equation eddy viscosity turbulence closure able to mimic the current response to waves from the laboratory experiments. They used it to study different wave-current alignments for shallow to deep water waves. Teles et al. (2013) used 3D Reynolds Averaged Navier-Stokes to evaluate how well three turbulent closure schemes could reproduce the conditions measured in laboratory by Umeyama (2005) and Klopman (1994). They concluded that the two first order models $(k-\varepsilon$ and $k-w)$ which rely on the eddy viscosity hypothesis do not reproduce laboratory results as well as the Reynolds Stress transport Model (RSM). Teles et al. (2013) however imposed a surface boundary condition to their first order models which forces the eddy viscosity to decrease towards the surface. This is contrary to what is imposed in the realistic simulations presented here where wave coupling leads to a surface enhancement of the eddy viscosity due to the input of wave induced TKE.

A fundamental discrepancy between the current work and the laboratory experiments, the theoretical work, and numerical studies discussed so far is in the relative magnitude of the currents and Stokes drift. While in the present study the tidal currents are much faster than the waves, they are of the same order of magnitude in the aforementioned studies. Furthermore, the current work focuses on deep water waves. By contrast, the laboratory experiments, which the previous studies aim to reproduce, featured intermediate depth waves with a ratio of wave height over depth of 0.24 and 0.1 respectively for Klopman (1994) and Umeyama (2005). Consequently only limited comparisons to previous laboratory and analytical works are possible. Numerical development may be necessary to improve the dynamics and kinematics of surface wave-current interactions but dedicated in situ surface current and turbulence profile measurements are also needed for validation.

\subsection{Comparison to Regional Coupled Modelling Studies}

Previous realistic coupled modelling studies, which do not include an RSM, reported no substantial impact of waves on currents. Closer inspection of published figures, however, reveals a wave induced change in currents of about $10 \%$ in two of them which is of the same order as those reported herein. 
These studies were conducted under much stronger wind conditions and weaker currents than those simulated here: one during wind-jet events with wind induced currents (Ràfols et al., 2019), the other in a tidal estuary (Osuna and Wolf, 2005). Using the COAWST modelling system for the north Ebro shelf, Ràfols et al. (2019) showed that the main impact of waves was on the water column stratification as waves inject turbulent kinetic energy and enhance surface stress thus deepening the mixed layer. Their results indicate differences in surface currents of up to $20 \mathrm{~cm} \mathrm{~s}^{-1}$ for currents ranging between 20 to less than 80 $\mathrm{cm} \mathrm{s}^{-1}$ under winds from 10 to $20 \mathrm{~m} \mathrm{~s}^{-1}$. Osuna and Wolf (2005) reported a daily mean differences of up to $10 \mathrm{~cm} \mathrm{~s}^{-1}$ during a strong west-southwesterly wave event under winds of $15-20 \mathrm{~m} \mathrm{~s}^{-1}$ in the shallow coastal areas (with depth around $50 \mathrm{~m}$ ) of the eastern Irish Sea where the current magnitude is around $1 \mathrm{~m} \mathrm{~s}^{-1}$. This is based on output from the coupled Proudman Oceanographic Laboratory Coastal-Ocean Modelling System-ProWAM framework. It is not possible to determine from the article how the observed enhancement relates to the relative alignment between the tidal currents and the waves.

The wave related processes found to be important in the Iroise Sea numerical experiments presented here are: the Stokes-Coriolis force which along with changes in vertical diffusion due to wave injection of TKE and surface stress alter surface currents. In the Irish Sea estuary, only the wave induced surface stress was reported to be key (Osuna and Wolf, 2005). Analysis of the cross-shelf momentum equation in the Ebro simulations revealed a main balance between the pressure gradient and the Coriolis term before wind events, although with wind speeds of $10 \mathrm{~m} \mathrm{~s}^{-1}$, with all other terms being an order of magnitude smaller (Ràfols et al., 2019). During wind-jet events in the Ebro region, the vortex force, pressure gradient, surface stress, and horizontal advection were found to be of the same order $4 \mathrm{~km}$ offshore, where the water is about $50 \mathrm{~m}$ deep. Further offshore, up to tens of kilometers, the vortex force and surface stress were shown to mostly balance the pressure gradient. In the macro tidal regime simulated in the Iroise Sea the wave effect on the horizontal advection and pressure gradient are negligible and under low wind conditions, the vortex force is found to be secondary to other wave processes. Its role in altering the currents and front position under stronger winds has yet to be investigated.

\subsection{Comparison to Remotely Sensed Measurements}

The partially coupled simulations reveal that advecting the temperature with the Lagrangian velocity without allowing feedbacks of wave mixing and the Stokes-Coriolis force leads to an eastward bias of the frontal position. The erosion of the temperature front associated with the wave mixing is also shown to be key and explains the westward shift of the front. Joint analysis of hourly satellite derived SST and $\mathrm{HF}$ radar derived currents at $48.75^{\circ} \mathrm{N}$ suggested that the Ushant front is not advected by the Stokes drift 
(Chevallier et al., 2014). Estimating the front position from advection based on HF radar derived currents during a period of moderate easterly winds $\left(5-10 \mathrm{~m} \mathrm{~s}^{-1}\right)$ led to a westward bias of tens of kilometers after a couple of days. A closer to real estimate of the temporal evolution of the front position by advection was only possible after removal of the Stokes bias (Ardhuin et al., 2009) from the current measurements. A small westward bias is still visible after the Stokes drift correction which may hint to the fact that it does not take into account the impact of surface gravity waves on the quasi-Eulerian currents. The present study suggests that for a more complete correction of the Stokes drift effect in HF radar measurements, the knowledge of the vertical gradients of the horizontal velocity is necessary as well as an estimate of the wave induced mixing. While the finding of Chevallier et al. (2014) may suggest that the surface temperature should be advected by the quasi-Eulerian velocity only, the authors ignore the impact of local tidally induced adiabatic mixing which maintains the frontal dynamics. As the tidal residual current in the region is to the north-east, pure advection would lead to an eastward drift of the front which overtime would end up on the coast. This is clearly not observed. The HF radar measurements give the currents within the top tens of centimeters of the water column while the surface frontal signature results from the dynamics of the top tens of meters. Advecting the front by Lagrangian HF radar observations thus overestimates the relative importance of the Stokes drift compared to other wave impacts (mixing) and leads to a front displacement disconnected from mixed layer dynamics.

\subsection{Limitations and Avenues to Improve the Coupled Framework}

The impacts of TKE production by Stokes drift and Langmuir turbulence were not included in the present study nor the previously mentioned coastal coupled modeling efforts. As the current horizontal resolution is too coarse to resolve Langmuir circulations (100 m - $1 \mathrm{~km})$ a parameterization would need to be included in the energy equations to take them into account. Several approaches can be envisioned based on previous studies (e.g. Kantha and Clayson, 2004; Harcourt, 2013). Langmuir turbulence has the potential to impact the water column deeper than wave breaking. Thus including it could lead to erosion of the temperature stratification on the offshore side of the front, further contribution to a offshore shift in its location. Another avenue for improving the current framework is to lift the hydrostatic constraint or to include the effects of the Stokes shear force on the pressure term (see Suzuki and FoxKemper, 2016). A higher vertical resolution would also be needed to properly resolve the Stokes shear. As it stands, using the Breivik et al. (2014) parameterization for the vertical Stokes drift profiles, the surface Stokes shear is underestimated. In the current framework, the surface current fed to the wave model is that of the top vertical mode. In situations of strong surface current shear it may be more appropriate to consider a vertically integrated current $(S u z u k i, 2019)$. The integration depth considered 
could further be wavelength dependent. This however would add considerable computations. Improving this parameterization, increasing the resolution, and applying the quasi-hydrostatic approach (Suzuki and Fox-Kemper, 2016) would increase the impact of the Stokes vortex force. Many uncertainties still surround the other parameterizations on which the framework relies. These include the source functions of the wave model which are used to derive the surface stress felt by the ocean $\left(\tau_{\text {surf }}\right)$ and wave to ocean energy flux $\left(\Phi_{o c}\right)$ as well as the boundary conditions of the turbulence closure scheme.

\subsection{Broader Implications}

The frontal displacement reaches up to $4 \mathrm{~km}$ within 1.5 days as waves propagating into the region grow. Under steady wave forcing one can expect the front to shift until a new equilibrium is reached and wave breaking induced mixing has eroded the surface velocity and temperature gradients. Frontal displacements can be observed repeatedly over periods of a couple of days in a 14 day run with realistic forcing. Note that for the conditions simulated in the present study, low wind and tidal dominated dynamics, the two-way coupling between ocean and waves does not give significantly different results than when forcing the ocean by wave model fields as long as tidal currents were used to produce the wave fields. This was tested by running the coupled framework allowing only wave fields from a stand-alone WW3 run to be sent to the ocean model hourly. Indeed, in the studied situation, the described changes in the (quasi-)Eulerian currents are too small to significantly alter the properties of the swell. Thus, in this particular case coupling is not necessary for either the wave or the ocean components. This may however not be the case in non tidally dominated environments where currents are weaker or under stronger winds or under short fetches as wind-seas are more sensitive to currents.

\section{Conclusions}

The current study shows how surface gravity waves alter the location of a surface tidal temperature front. In accordance to previous theoretical and laboratory works, the coupled modelling framework reproduces a reduction (increase) of the surface quasi-Eulerian currents when they follow (oppose) waves. In a situation of mainly eastward propagating waves as in the present case study, the front is advected westwards by up to $4 \mathrm{~km}$ due to a reduction in the flooding tide and an increase in the ebb flow. This counter-intuitive impact opposes and over compensates the advection effect of Stokes drift around the front. Note that the situation studied here is not strongly forced by winds and tidal currents are much stronger than the wave induced drift. Yet the impact of waves is clearly detectable. The processes at play 
are summarized in the diagram in Figure 15. Here horizontal ocean and wave variability on either side of the front are neglected as the resolution is not yet sufficient to properly reproduce them. In the current coupled framework, the main wave dynamics behind the changes in currents are the vertical mixing and the Stokes-Coriolis force which leads to a transport that opposes the Stokes drift. Including wave physics is seen to increase the vertical mixing at the sea surface due to the TKE injection related to wave breaking. This increase in vertical mixing results in a reduction of the surface vertical gradient of the current which is positive for both flood and ebb tides leading to the observed changes in quasi-Eulerian currents. Within the studied environmental setting (strong tidal currents, moderate waves and winds) it appears that only forcing the turbulence closure with a surface wave breaking energy flux is sufficient to reproduce the wave impact produced by the fully coupled framework. In environments which are much more sensitive to the local wind conditions such as upwelling systems or under extreme conditions feedback between the ocean and the waves will have to be taken into account. Before a definite conclusion can be reached on which coupling aspects are necessary for operational use in coastal seas, the coupled model framework will have to be improved further. Both the horizontal and vertical resolutions need to be increased and further development is needed to improve the representation of the Stokes shear and Stokes vortex force and include Langmuir turbulence. Nevertheless, the present study highlights the importance of taking wave-current interactions into account even in environments where the background flow is an order of magnitude stronger than the Stokes drift. Thus, waves should be considered in dispersion studies even in tidally forced coastal seas. The enhanced surface mixing resulting of injection of TKE from wave breaking can significantly vertically homogenize surface tidal currents even under moderate winds. In altering the surface advection, waves can lead to displacements of fronts. Valid in a tidally forced environment, the processes described herein are likely more important where surface currents are weaker. Susceptible to influence the position of any surface temperature front, wave-current interactions could be important in dictating the offshore extent of upwelling systems. The coupled model framework exploited herein would allow studying coupling dynamics and energetics in a wide variety of coastal settings through analysis not only of state variables but also of the terms of the temperature, current, and energy equations.

\section{Acknowledgements}

The AMICO project was co-funded by Copernicus, the French ministry for the Environment, Energy and the Sea, CNRS-INSU and LEFE-GMMC. SEB is currently supported by a postdoctoral grant from the Centre National d'Études Spatiales (CNES). Numerical simulations were performed on Ifremer HPC facilities DATARMOR of the Pôle de Calcul Intensif pour la Mer (PCIM) (http://www.ifremer.fr/pcim). 
681 The presented coupled framework is based on a prototype wave-ocean coupled model for monochromatic 682 waves developed by A.-C. Bennis from which an idealistic test case can be found in Bennis et al. (2011). ${ }_{683}$ The authors would like to thank three anonymous reviewers as well as N. Suzuki for their comments that 684 allowed for an improved manuscript. 
(a)

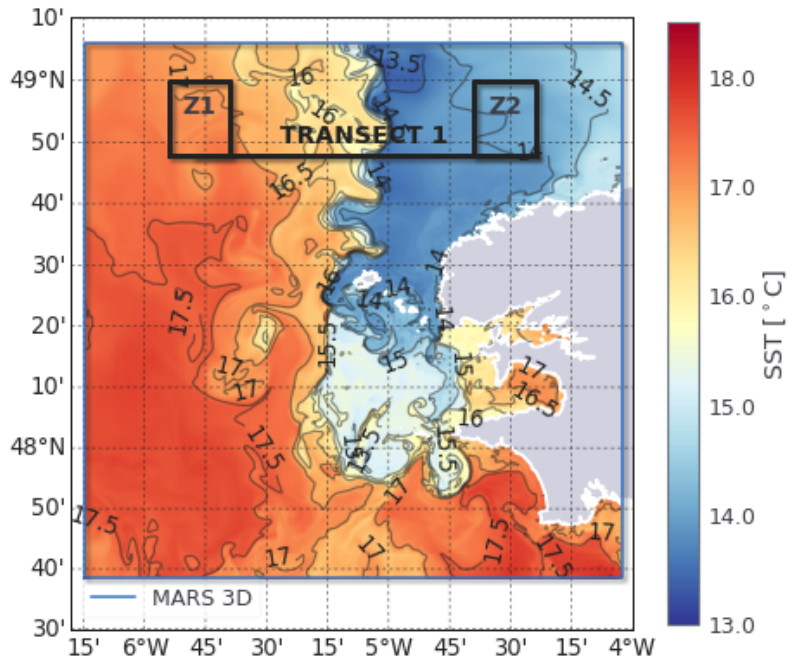

(b)

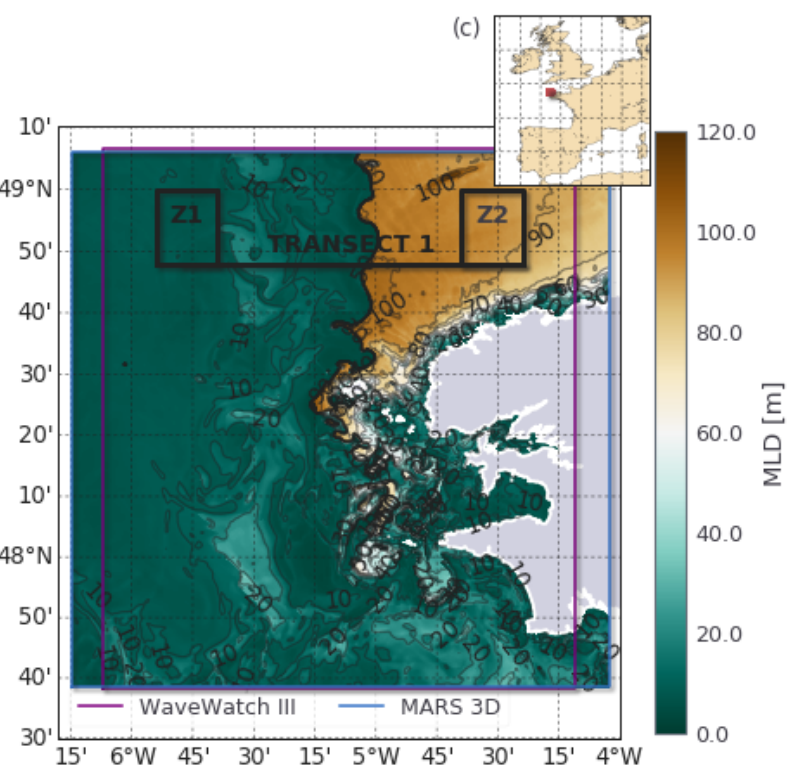

Figure 1: Maps of (a) the sea surface temperature (SST) and (b) the mixed layer depth (MLD) from the stand-alone MARS3D ocean model run for 02 September at 1200 UTC. The map in (c) shows the location of the study region denoted by a red box off the coast of Brittany, France. Also shown in (a) and (b) are the model domains and the regions of interest considered in this study. 
WAVEWATCHIII

Mean Period $\left(T_{m 1}\right)$

Significant Wave Height $\left(H_{s}\right)$

Wave induced pressure (J)

Stokes transport $\left(T_{s, x} T_{s, y}\right)$

Surface Stokes drift $\left(u_{s, 0} v_{s, 0}\right)$

Surface stress $\left(\tau_{a w, x}, \tau_{a w, y}\right)$

Wave-ocean momentum flux $\left(\tau_{\text {wo,x }} \tau_{\text {wo,y }}\right)$

Bottom wave dissipation stress $\left(\tau_{\mathrm{bw}, \mathrm{x}}, \tau_{\mathrm{bw}, \mathrm{y}}\right)$

Near-bottom rms wave velocity $\left(u_{b w}, v_{b w}\right)$

Surface wave breaking energy flux $\left(\phi_{o c}\right)$

Bottom wave dissipation energy flux $\left(\phi_{b b l}\right)$
3

Sea Surface Height $(\zeta)$

Sea Surface Currents $\left(\hat{\mathrm{u}}_{0}, \hat{v}_{0}\right)$

17

MARS3D

Figure 2: Diagram showing the variables exchanged between the ocean (MARS3D) and wave (WAVEWATCHIII) models during coupling. 
(a)

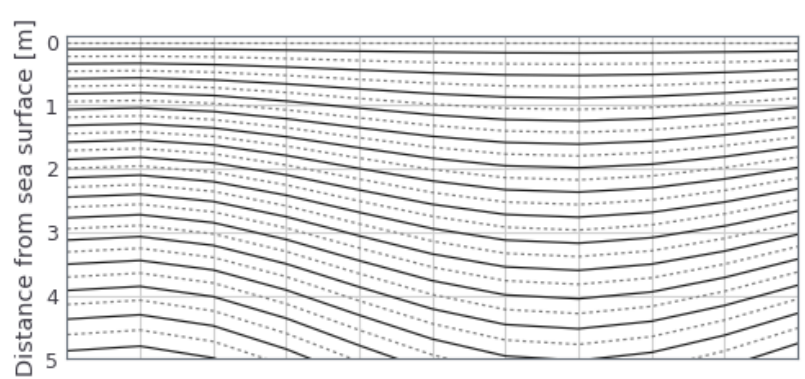

(b)

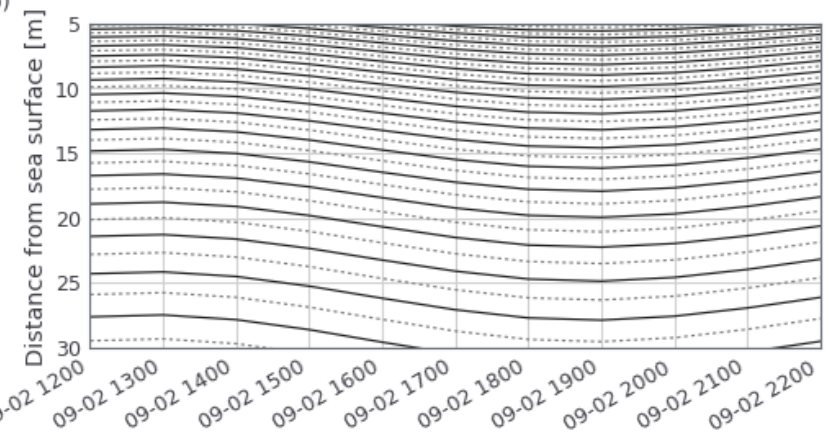

Figure 3: Time series over a tidal cycle of the vertical levels resolved in MARS3D (a) from the sea surface to $5 \mathrm{~m}$ and (b) from 5 to $30 \mathrm{~m}$ below the sea surface. The solid lines represent levels at which the velocities, temperature, and salinity are evaluated, the dashed lines represent the mid-levels at which the $k-\varepsilon$ equations are evaluated. 

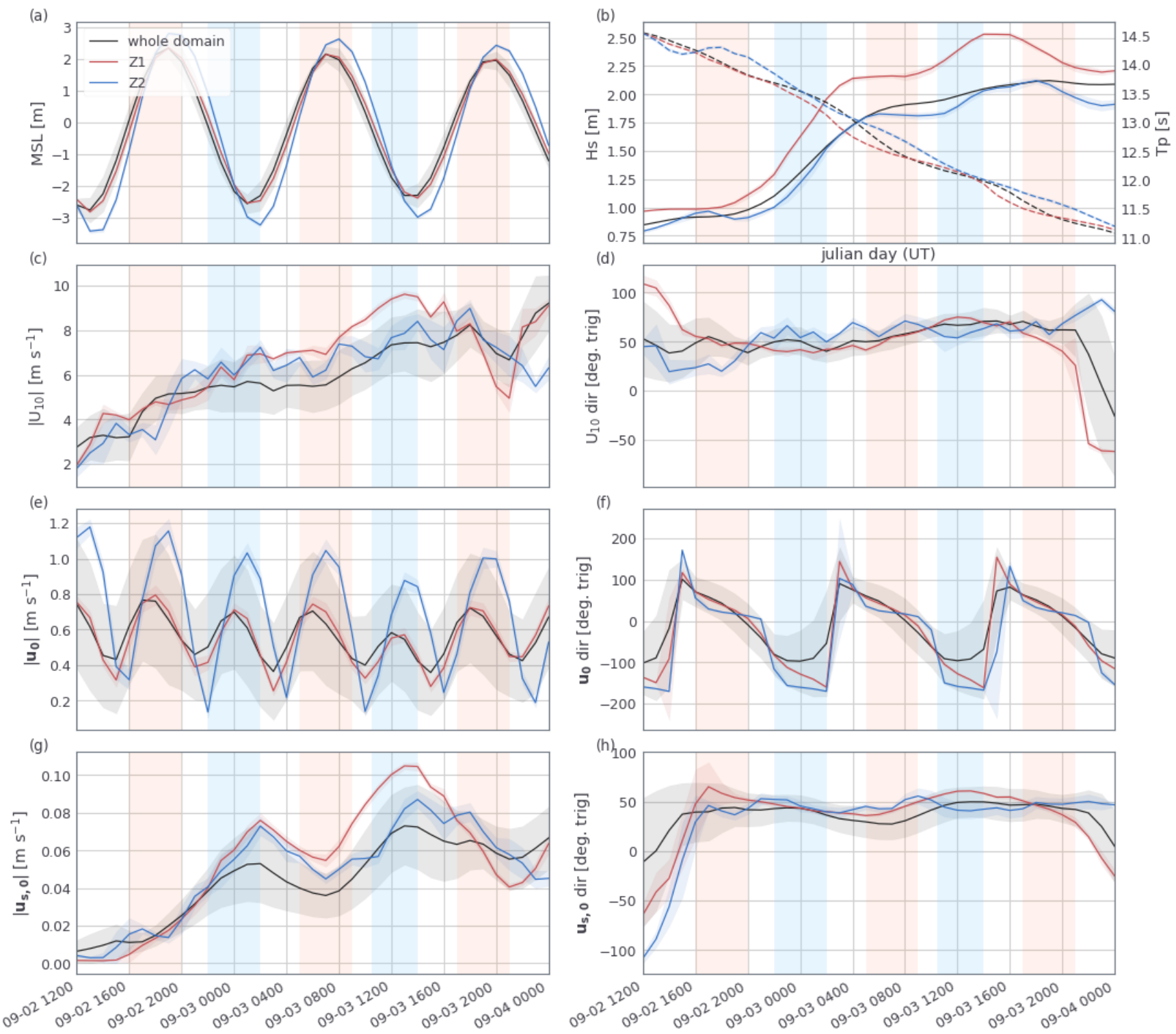

Figure 4: Time series of (a) the mean sea level, (b) the significant wave height and period of waves at the spectral peak, (c) the 10-m wind speed, (d) the direction of the 10-m wind in trigonometric convention, (e) the surface current speed from the stand-alone MARD3D run, (f) the direction of the surface current from the stand-alone MARD3D run in trigonometric convention, (g) the intensity of the surface Stokes drift, and (h) the direction of the surface Stokes drift in trigonometric convention. The black line represents an average over the whole domain while the blue and red lines are the averages in $\mathrm{Z} 1$ (warm, stratified side of front) and Z2 (cold, mixed side of front), respectively. Shadings around the mean curves correspond to \pm one standard deviation. The light blue and coral shadings demarcate periods of wave-opposing and wave-following currents, respectively. 
(a)

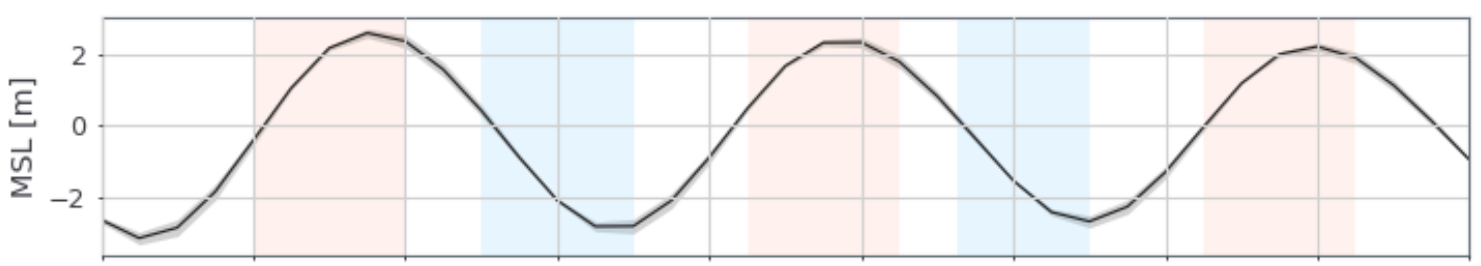

(b)

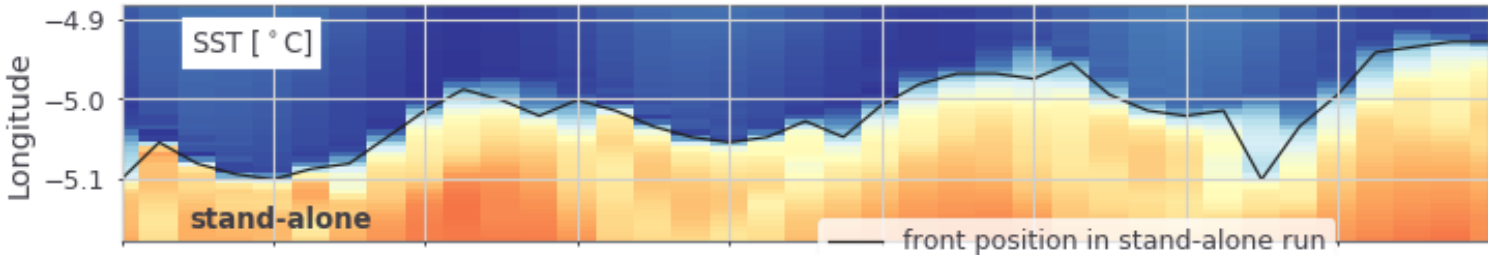

(c)

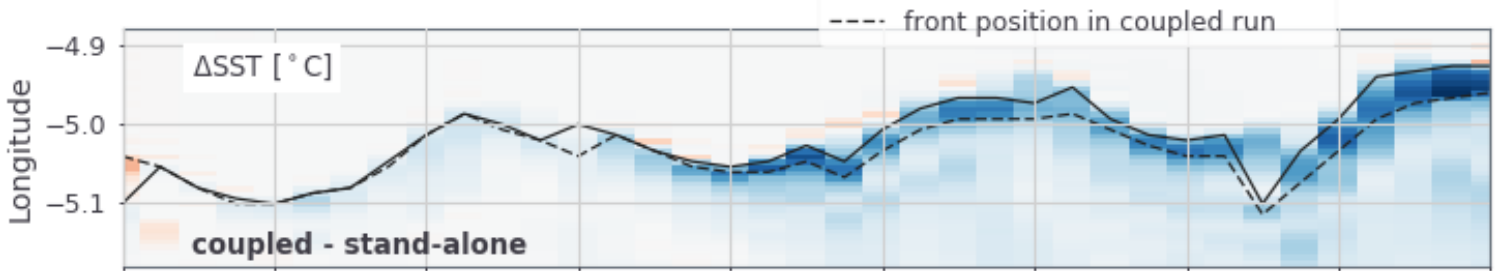

(d)

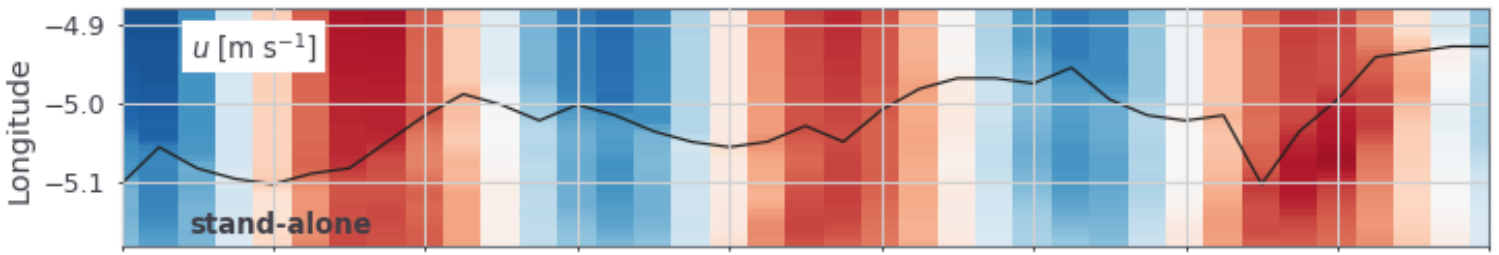

(e)
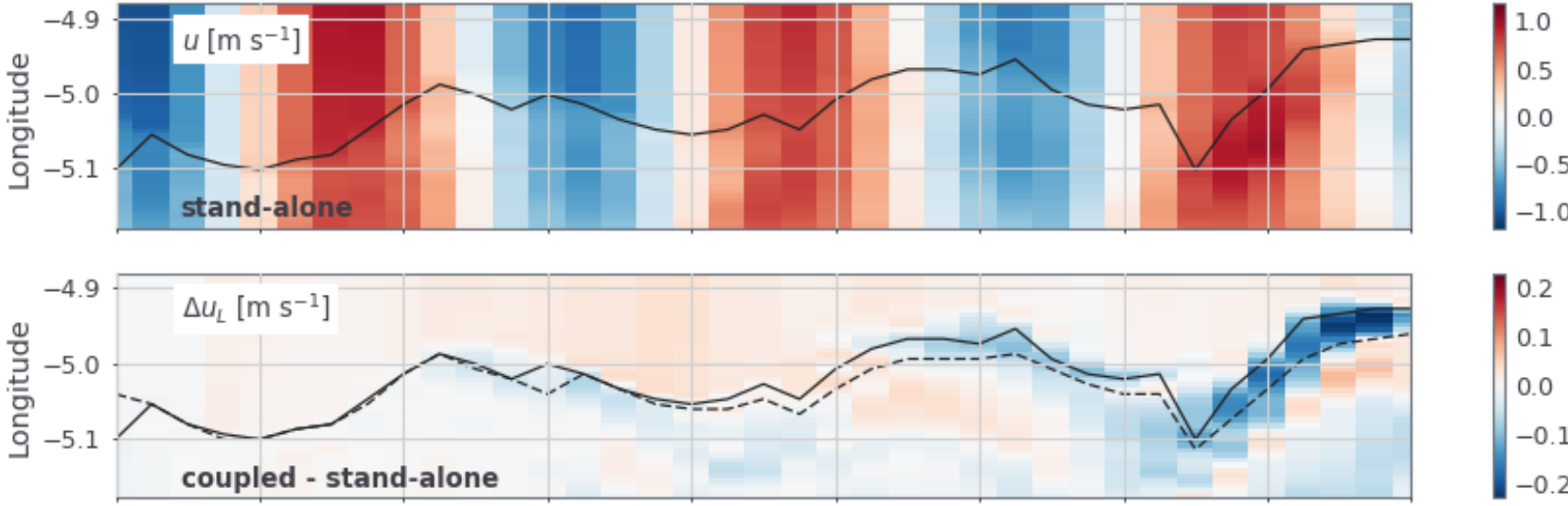

(f)

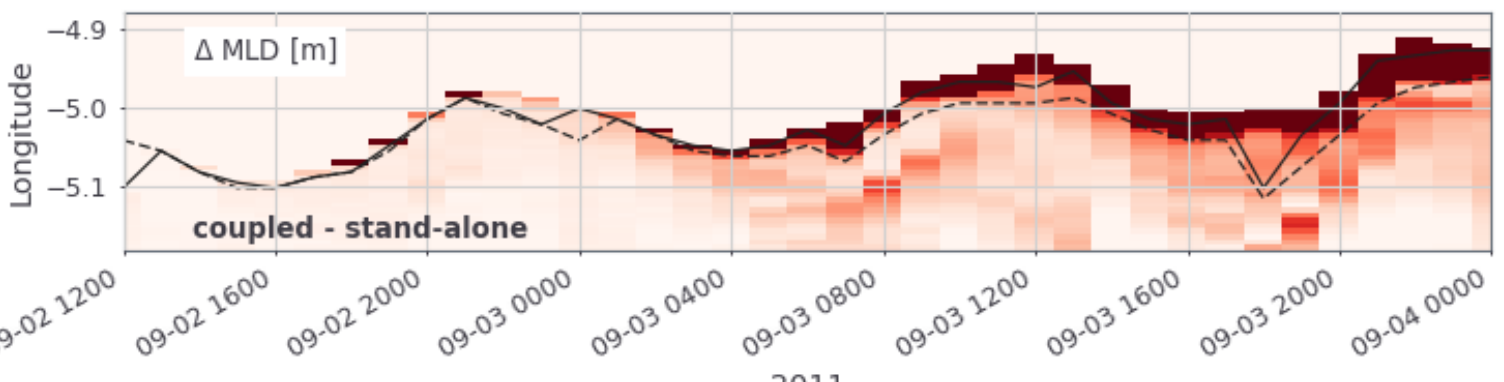

Figure 5: (a) Time series of the mean sea level along the transect at $48.8^{\circ} \mathrm{N}$, between 5.2 and $4.8^{\circ} \mathrm{W}$ (see Fig. 1), and Hovmöller plots at $48.8^{\circ} \mathrm{N}$ of (b) the SST from the stand-alone MARS3D run, (c) the SST difference between the coupled MARS3D-WW3 and stand-alone MARS3D runs, (d) the zonal surface velocities from the stand-alone MARS3D run, (e) the difference in zonal surface tracer advecting velocities between the coupled MARS3D-WW3 and stand-alone MARS3D runs, and (f) the difference in mixed layer depths between the coupled MARS3D-WW3 and stand-alone MARS3D runs. The black lines indicate the position of the surface SST front in the stand-alone (solid) and coupled (dashed) runs. The light blue and coral shadings in (a) demarcate periods of wave-opposing and wave-following currents, respectively. 
(a)

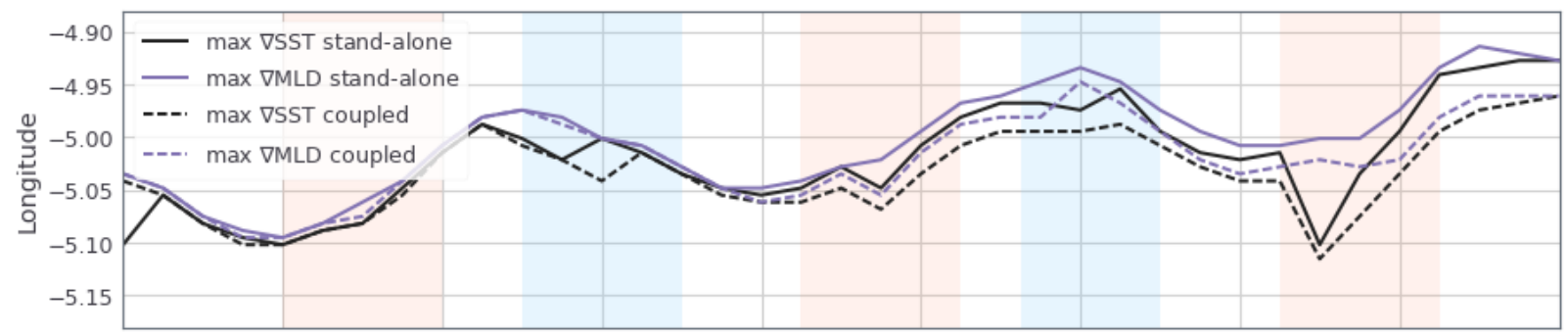

(b)

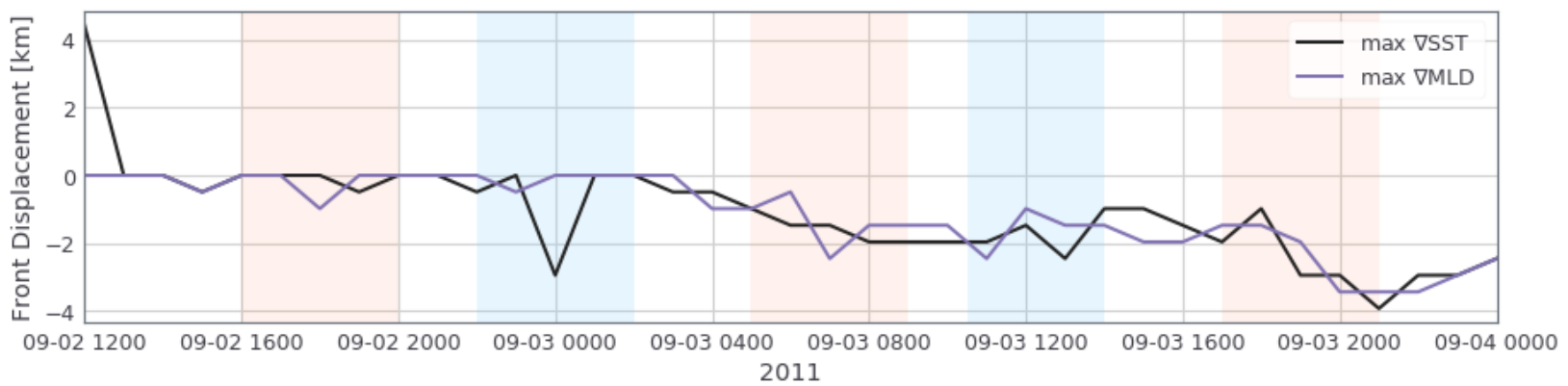

Figure 6: Time series at $48.8^{\circ} \mathrm{N}$ of (a) the position of the Ushant front based on the maximum gradient in SST (black) and MLD (purple) and (b) the displacement of the front in km based on maximum gradient in SST (black) and MLD (purple). In (a) solid lines correspond to the stand-alone MARS3D run and dashed ones to the coupled MARS3D-WW3 run. The light blue and coral shadings demarcate periods of wave-opposing and wave-following currents, respectively. 
(a)

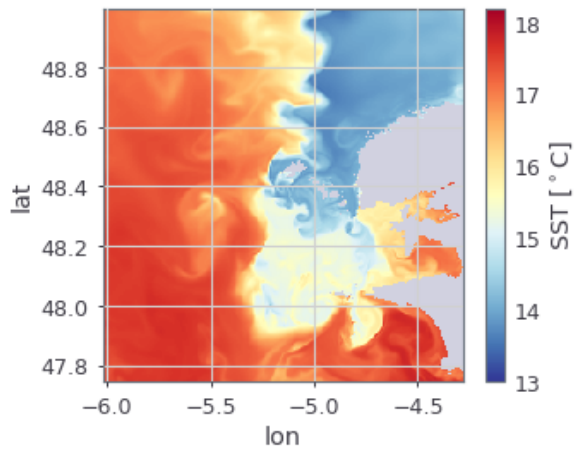

(b)

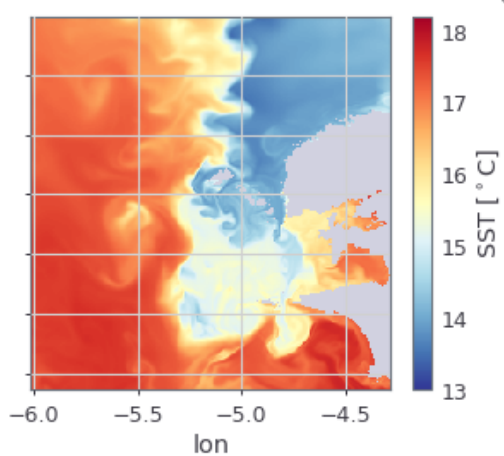

(c)

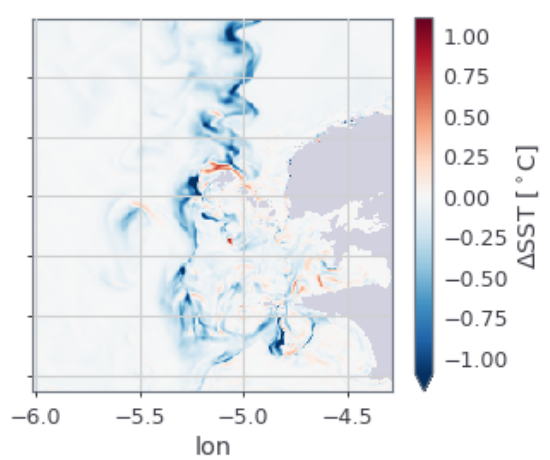

Figure 7: Maps of the sea surface temperature from (a) the stand-alone MARS3D run and (b) coupled MARS3D-WW3 run as well as (c) difference between the coupled and the stand-alone runs for 03 September 2011 at 1300 UTC. 

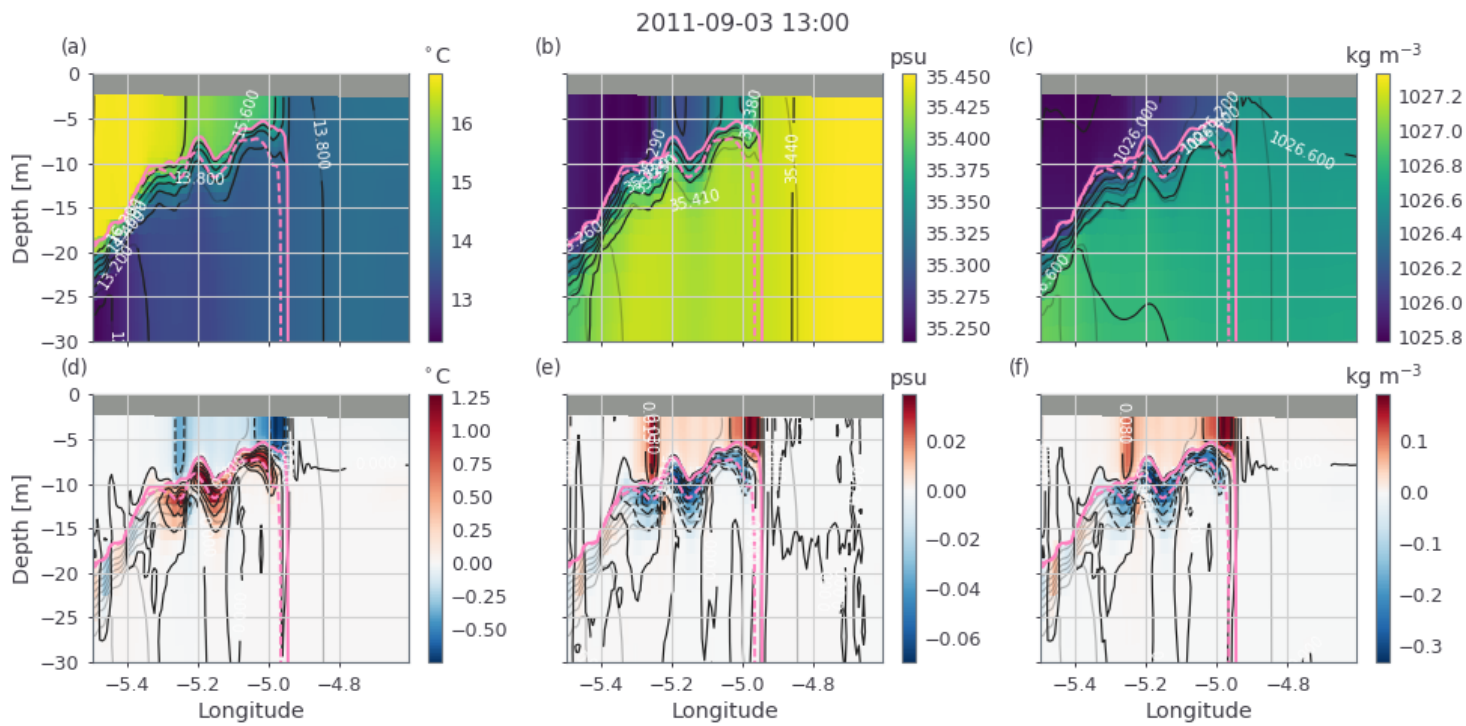

Figure 8: Depth vs. longitude transects at $48.8^{\circ} \mathrm{N}$ of (a) the potential temperature, (b) the salinity, and (c) the density from the stand-alone MARS3D ocean model run. Transects of the difference at $48.8^{\circ} \mathrm{N}$ in (d) potential temperature, (e) salinity, and (f) density between the coupled MARS3D-WW3 run and the stand-alone MARS3D run. The light grey contours in (b) through (f) are of the potential temperature of the stand-alone run. In pink the mixed layer depth of the stand-alone (solid) and coupled (dashed) runs. 
(a)

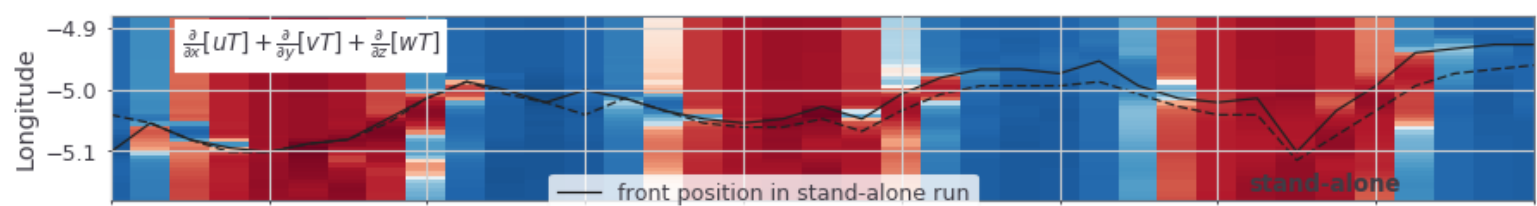

(b)

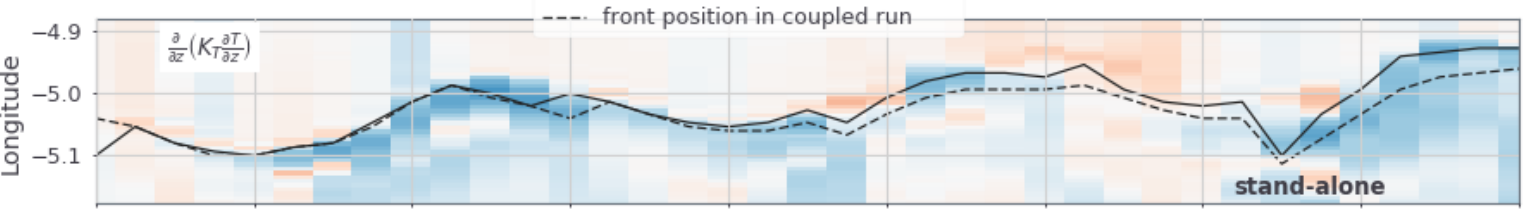

(c)

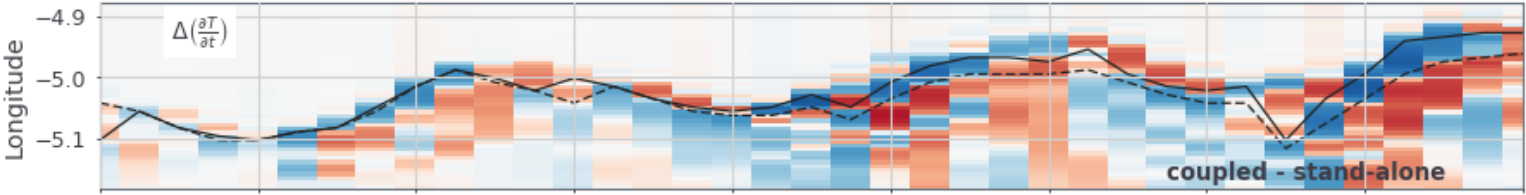

(d)

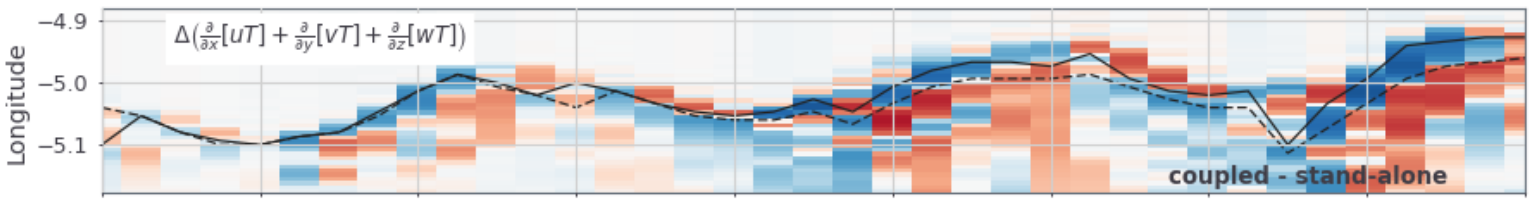

(e)

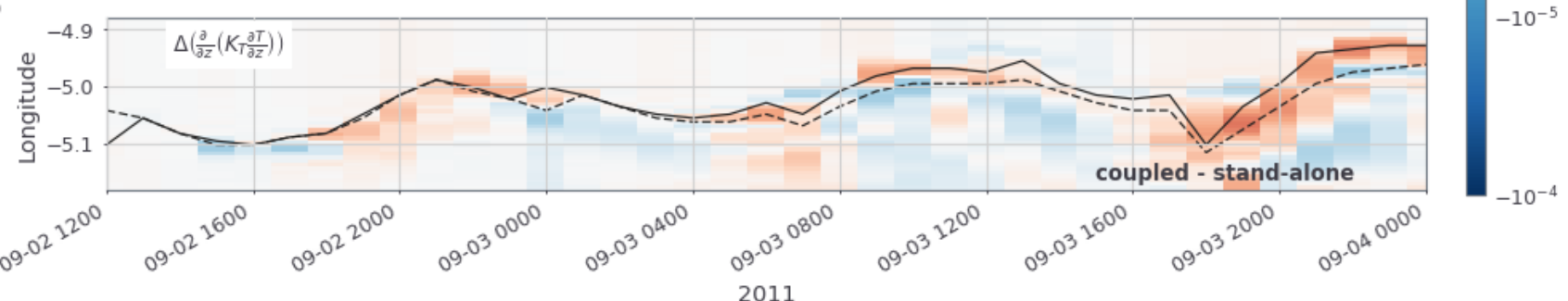

Figure 9: Hovmöller plots at $48.8^{\circ} \mathrm{N}$ of (a) the advection and (b) the vertical diffusion of the surface temperature diagnosed from the stand-alone MARS3D run, as well as of the differences (coupled - stand-alone) of (c) the temporal trend, (d) the advection, and (e) the vertical diffusion of the surface temperature. The black lines indicate the position of the SST front in the stand-alone (solid) and coupled (dashed) runs. 

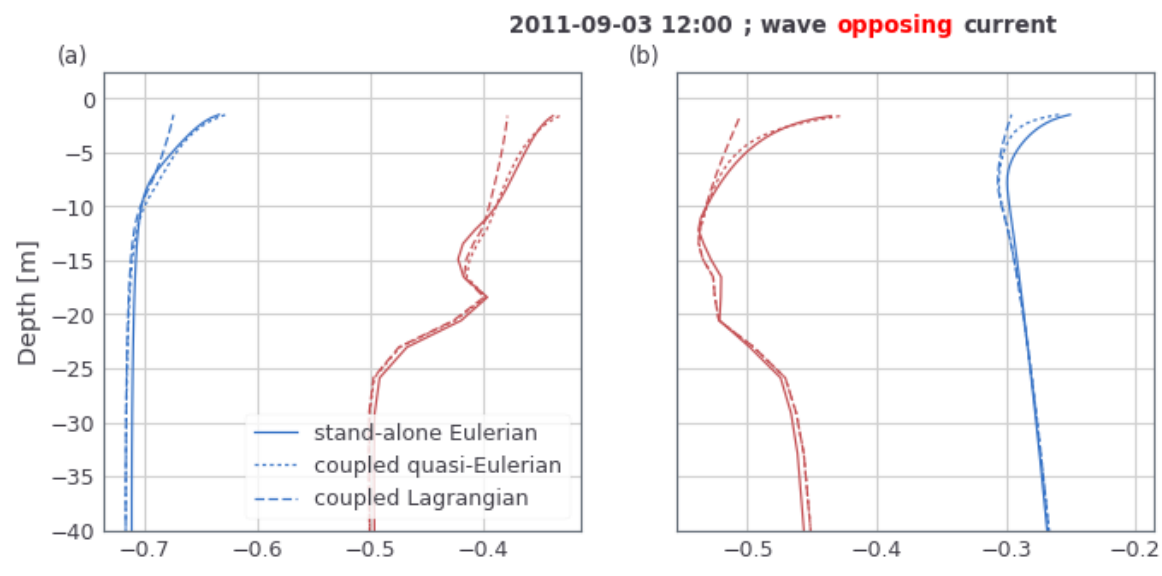

2011-09-03 18:00 ; wave following current

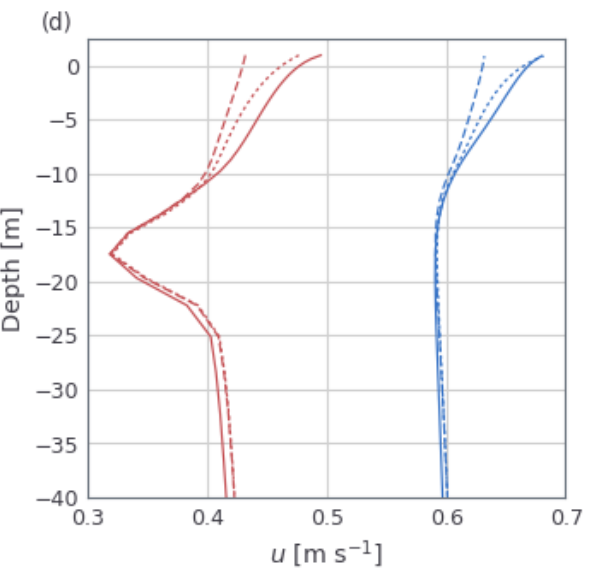

(e)

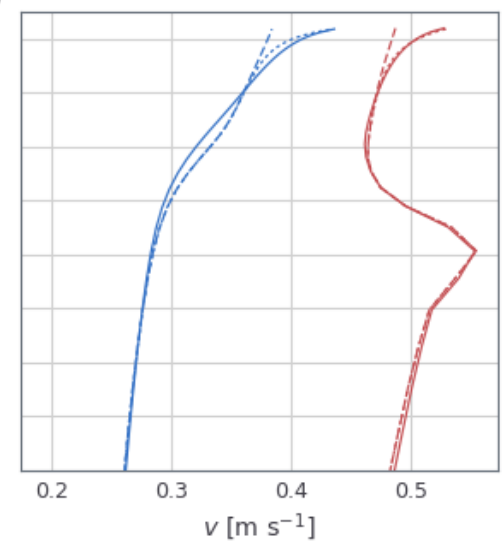

(c)

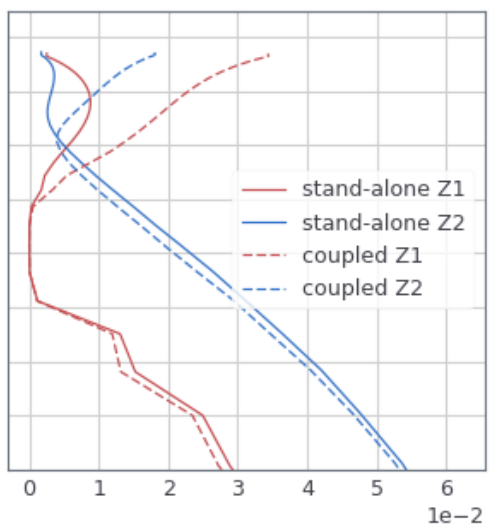

(f)

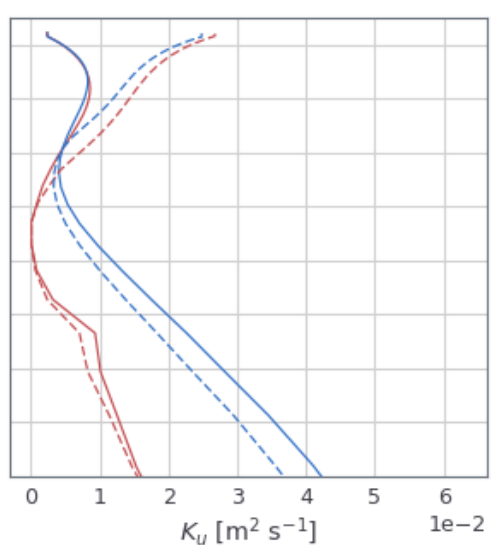

Figure 10: Vertical profiles of (a, d) the zonal and (b, e) the meridional components of the (quasi-)Eulerian and Lagrangian (dot dashed lines) velocities, as well as (c,f) the tidal eddy viscosities for two periods: 2011-09-03 12:00 (top row) corresponds to a period when waves oppose the tidal flow and 2011-09-03 18:00 (bottom row) to a period where wave and tidal currents are aligned. The red and blue lines correspond to averages within Z1 (warm, stratified side of front) and Z2 (cold, mixed side of front), respectively. Solid lines are from the stand-alone MARS3D run and dashed lines from the coupled MARS3D-WW3 run. 
(a)

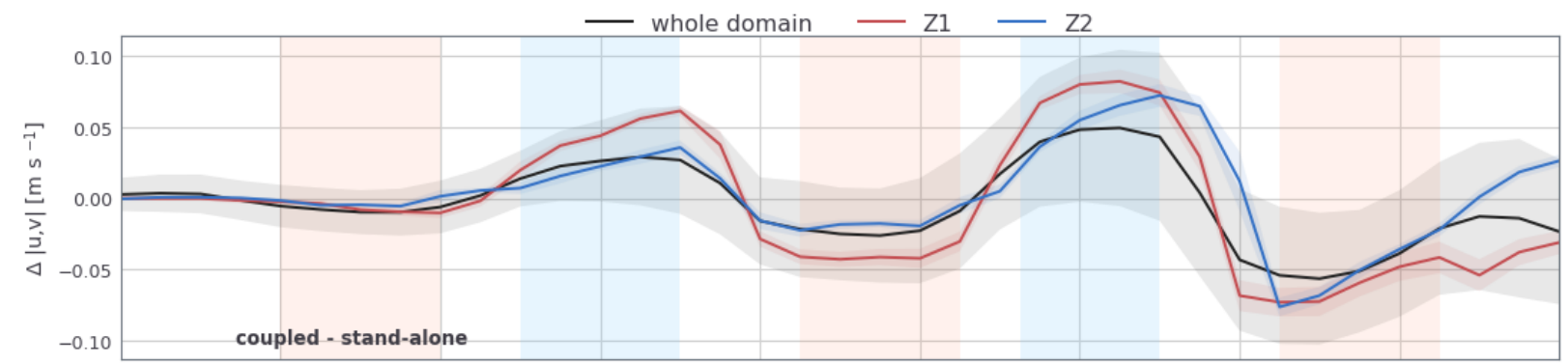

(b)

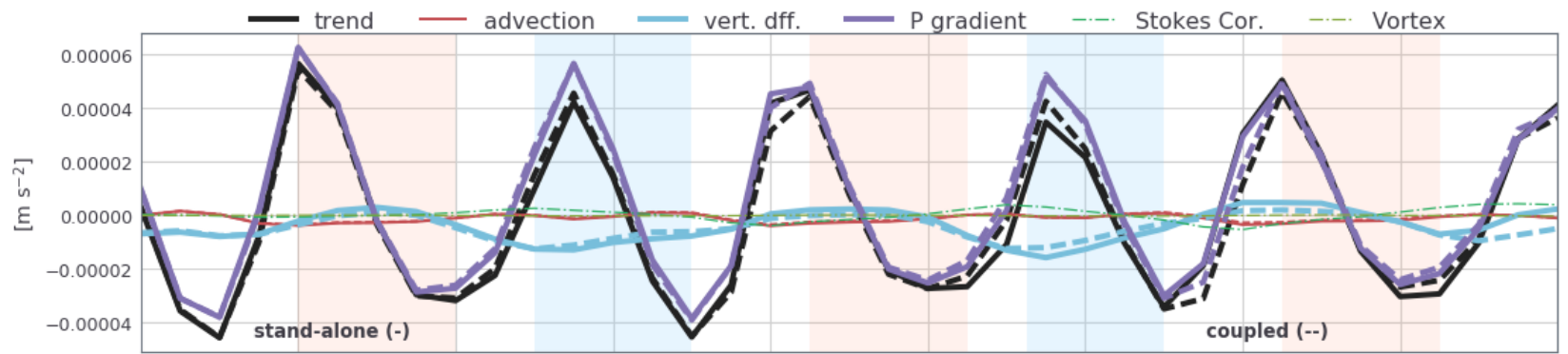

(c)

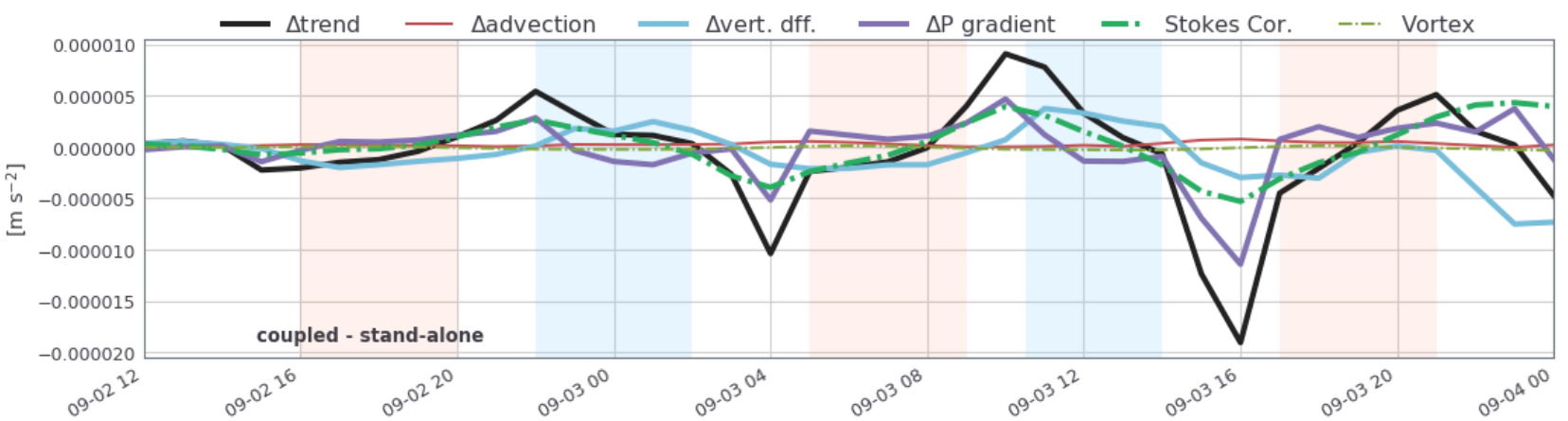

Figure 11: Time series of (a) the differences in the (quasi-)Eulerian surface current magnitude, (b) the terms of the equation for the surface current magnitude (Eqs. 1 and 8 transformed using Eq. 17) averaged over the whole domain, and (c) the differences (coupled - stand-alone) of the terms shown in (b). Note that the horizontal diffusion terms and the wave Bernoulli pressure head are omitted as negligible. In (a) the black line represents an average over the whole domain while the blue and red lines are the averages in Z1 (warm, stratified side of front) and Z2 (cold, mixed side of front), respectively. Shadings around the mean curves in (a) correspond to \pm one standard deviation. In (b) solid lines correspond to the output of the stand-alone run and dashed lines to the coupled run. Wave related terms are represented by the dot-dashed lines. The light blue and coral shadings demarcate periods of wave-opposing and wave-following currents, respectively. 
(a)

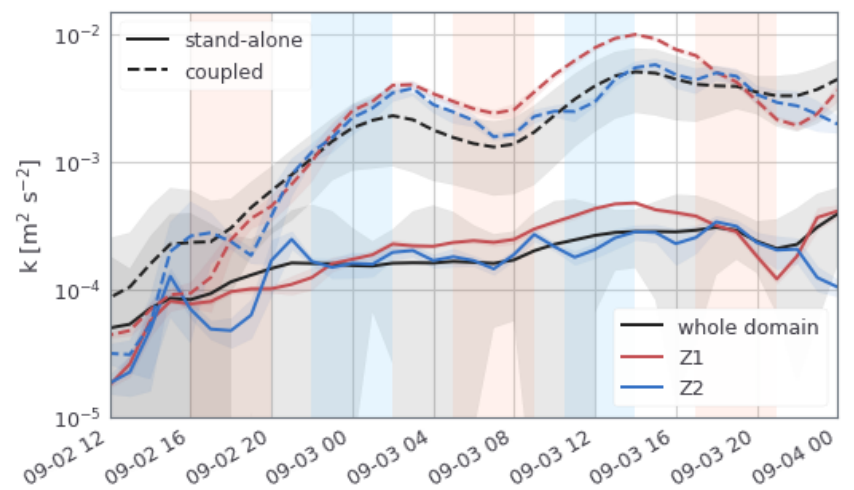

(b)

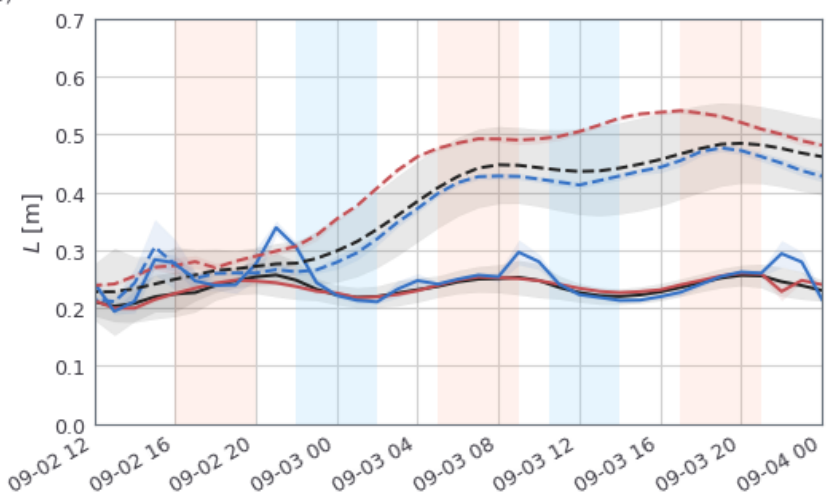

Figure 12: Time series of the near surface (a) turbulent kinetic energy and (b) turbulent length scale averaged over the whole domain (black), Z1 (red), and Z2 (blue) for the stand-alone (solid) and coupled (dashed) MARS3D runs. Shading around the curves represent \pm one stansdard deviation. The light blue and coral shadings demarcate periods of wave-opposing and wave-following currents, respectively. 

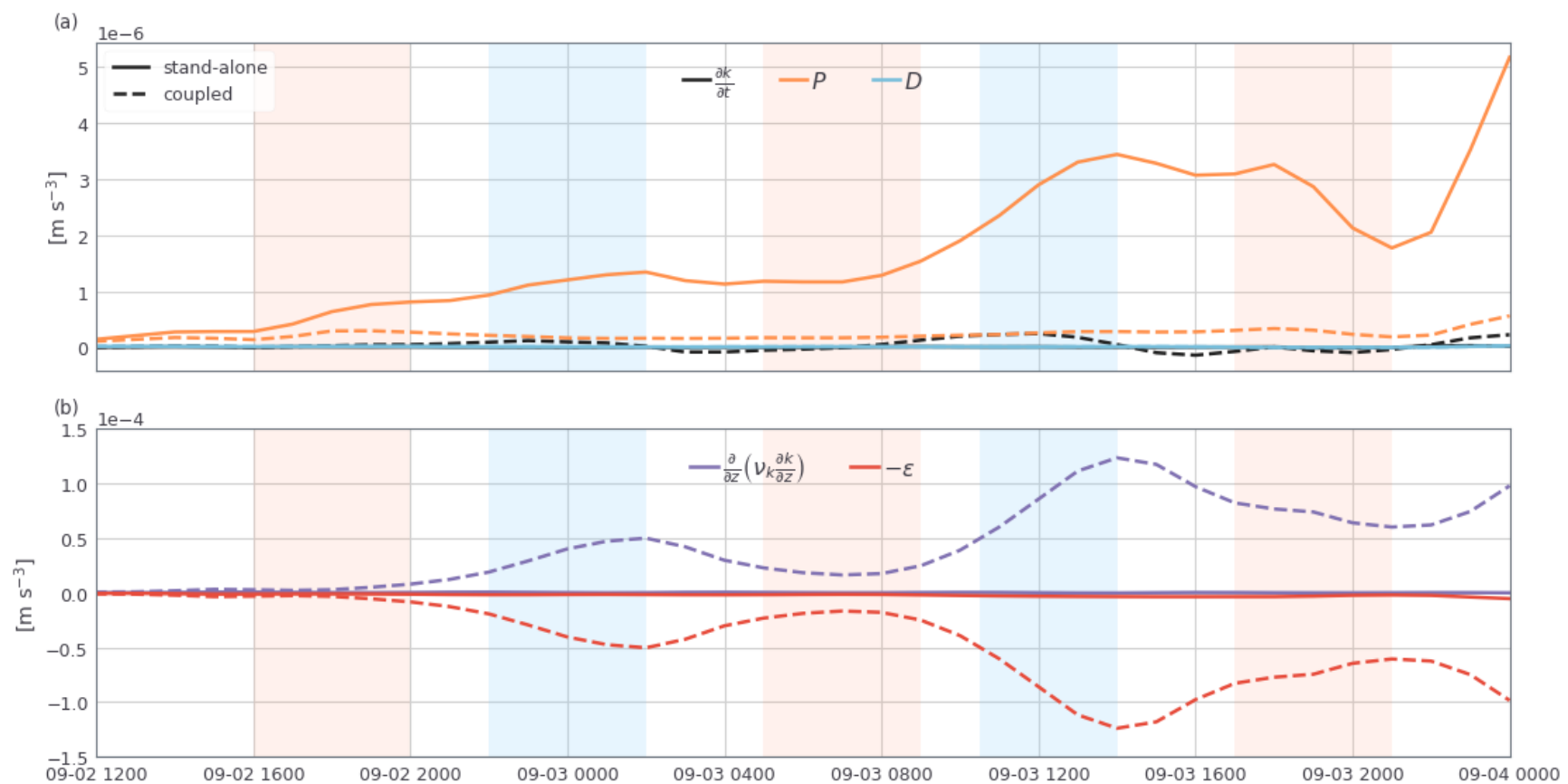

Figure 13: Time series of near-surface terms of the turbulent kinetic energy equation for the stand-alone (solid lines) and coupled (dashed lines) MARS3D runs averaged over the whole domain. Shown in (a) the trend (black), the shear production (orange), the buoyancy production (light blue), (b) the vertical diffusion (purple), the turbulent kinetic energy dissipation rate (red). The light blue and coral shadings demarcate periods of wave-opposing and wave-following currents, respectively. 

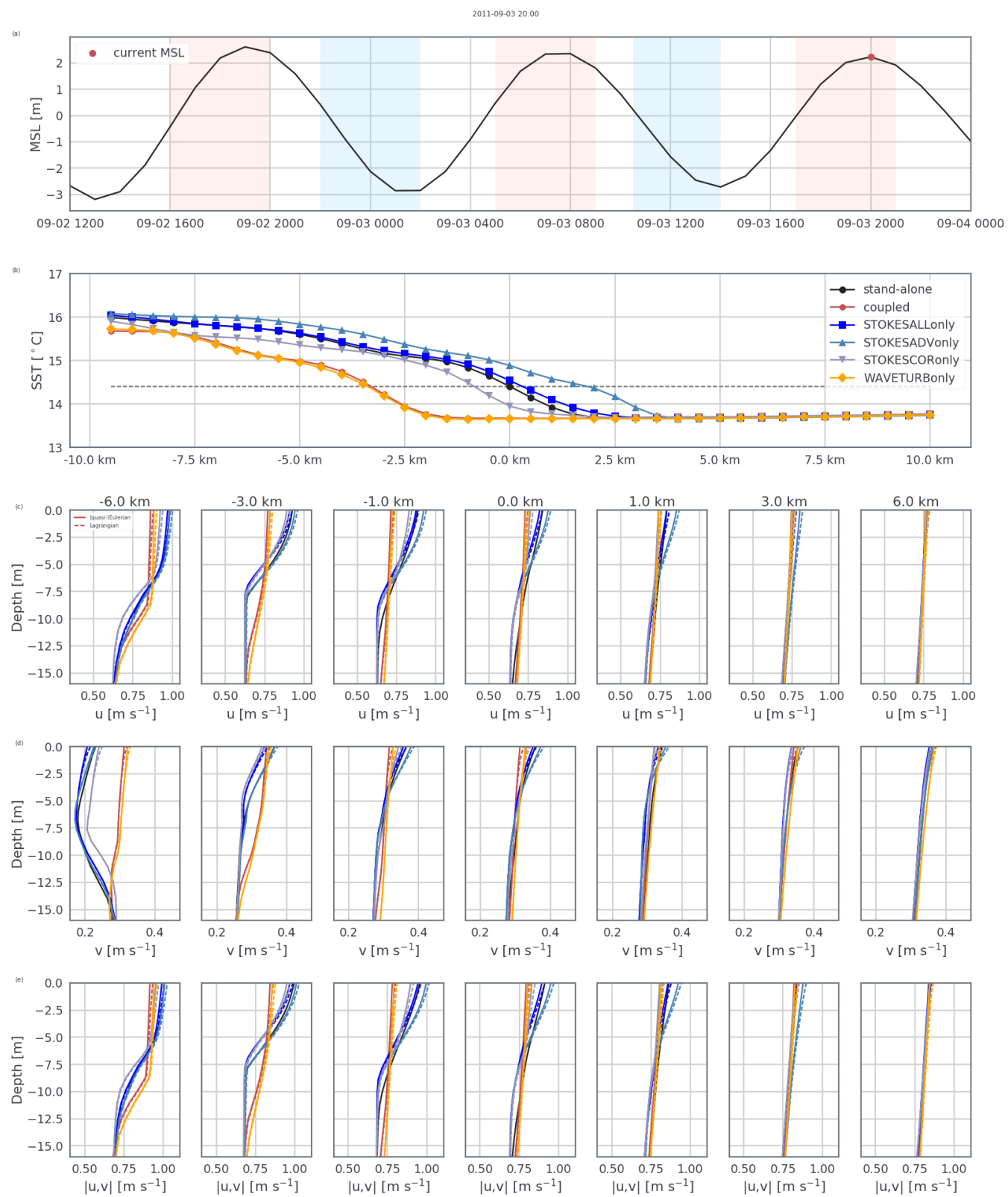

Figure 14: (a) Time series of the mean sea level putting the chosen snapshot (2011-09-03 2000 UTC, red dot) into the tidal context. (b) Across front SST transects on 2011-09-03 2000 UTC centered around grid point nearest to the $14.7^{\circ} \mathrm{C}$ isotherm (grey dashed line) in the stand-alone run. Vertical profiles on 2011-09-03 2000 UTC at several location (0 $\pm 1,3$, and $6 \mathrm{~km}$ from the grid point nearest to the $14.7^{\circ} \mathrm{C}$ isotherm in the stand-alone run) within the frontal region of (c) the zonal, and (d) the meridional components of the (quasi-)Eulerian (solid) and Lagrangian (dashed) currents as well as (e) their module. The light blue and coral shadings in (a) demarcate periods of wave-opposing and wave-following currents, respectively. 


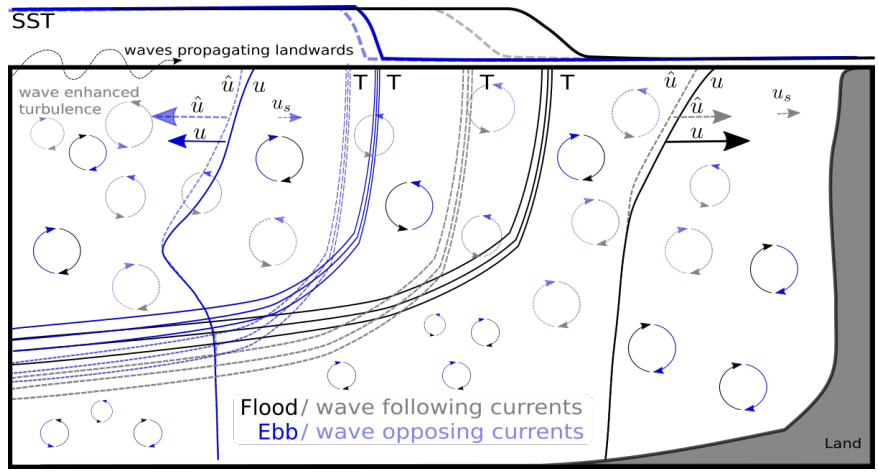

Figure 15: Diagram illustrating the impacts of shore-wards propagating surface gravity waves on a tidal front and currents. Solid lines represents wave free conditions and dashed lines show the wave induced changes for wave-following/flood (black) and wave-opposing/ebb (blue) currents. 


\subsection{Supplementary material}

\section{Supplementary Figure S1 is the full depth version of Figure 8.}

Supplementary Figures S2 and S3 show the terms of the current equation for the zonal and meridional components, respectively, similar to Figure 11.
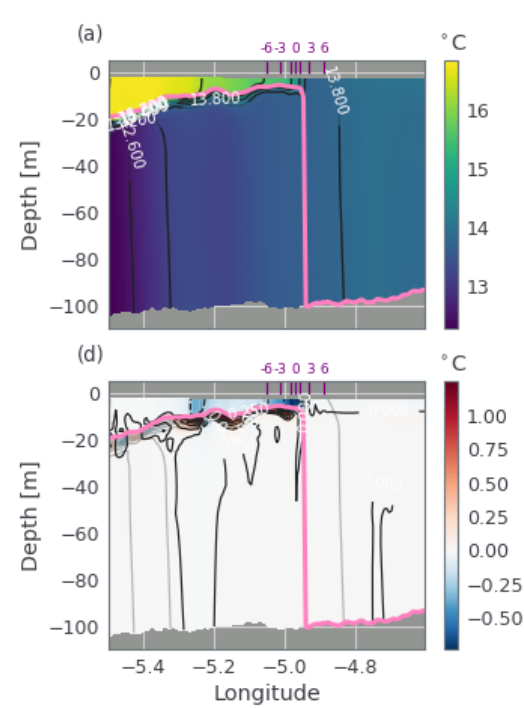
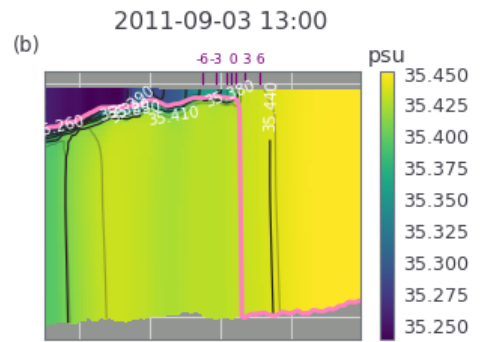

(e)

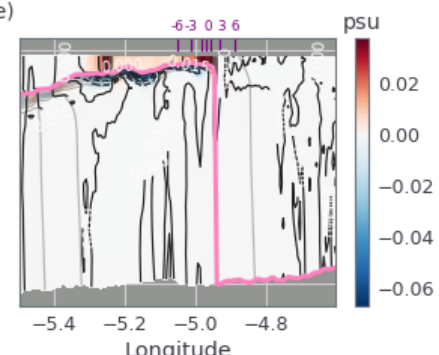

(c)

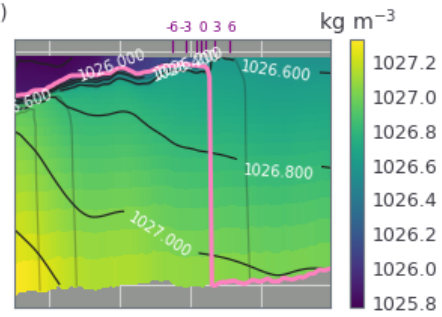

(f)

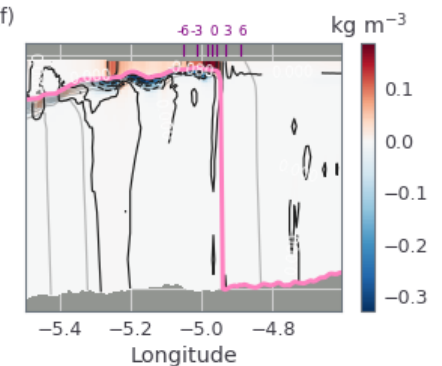

S 1: Depth vs. longitude transects at $48.8^{\circ} \mathrm{N}$ of (a) the potential temperature, (b) the salinity, and (c) the density from the stand-alone MARS3D ocean model run. Transects of the difference in (d) potential temperature, (e) salinity, and (f) density between the coupled MARS3D-WW3 run and the stand-alone MARS3D run. The light grey contours in (b) through (f) are of the potential temperature from the stand-alone run. In pink the mixed layer depth of the stand-alone MARS3D run. Also shown are the location of the profiles plotted in Fig. 14 indicated by the purple ticks at the top of each panel. The number indicates the distance from the 14.7isotherm in the stand-alone run. 
(a)

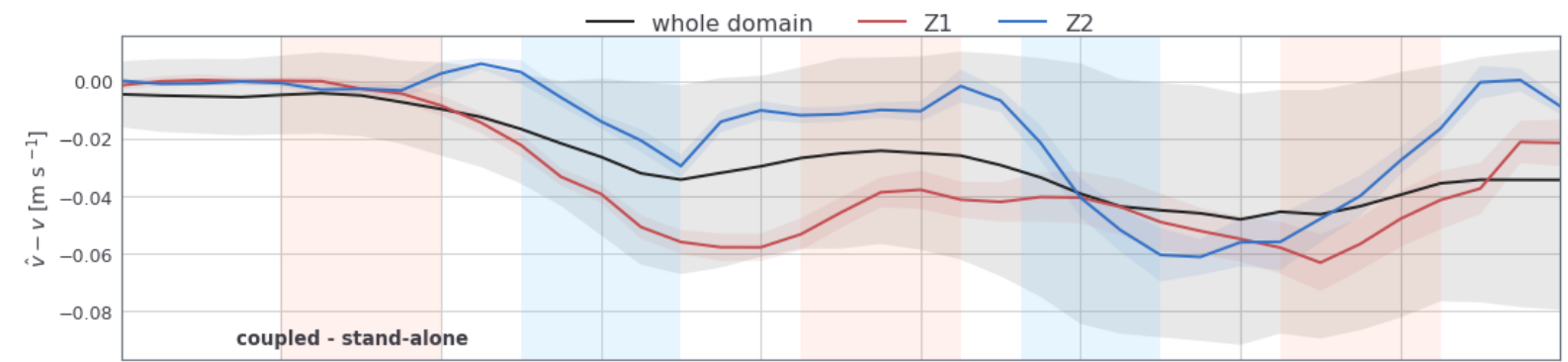

(b)

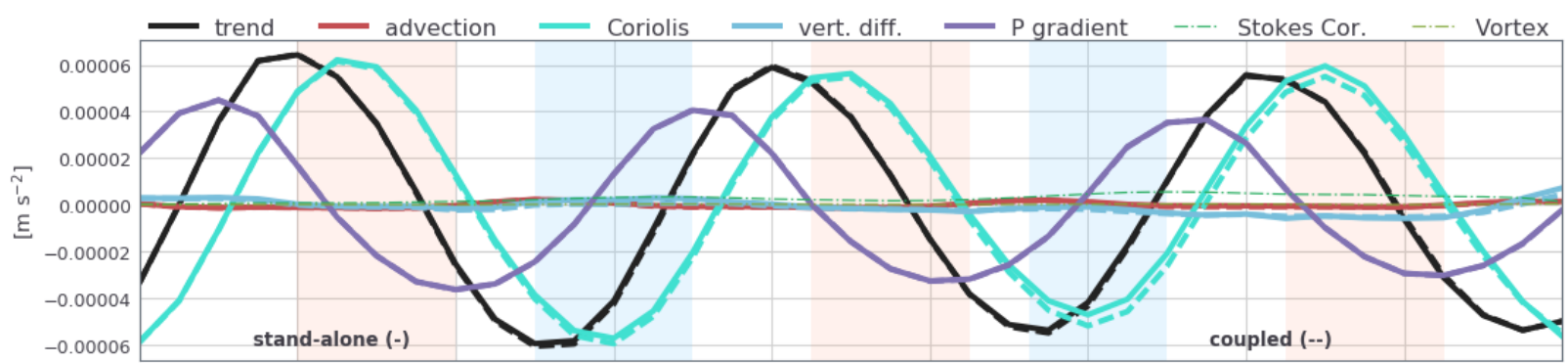

(c)

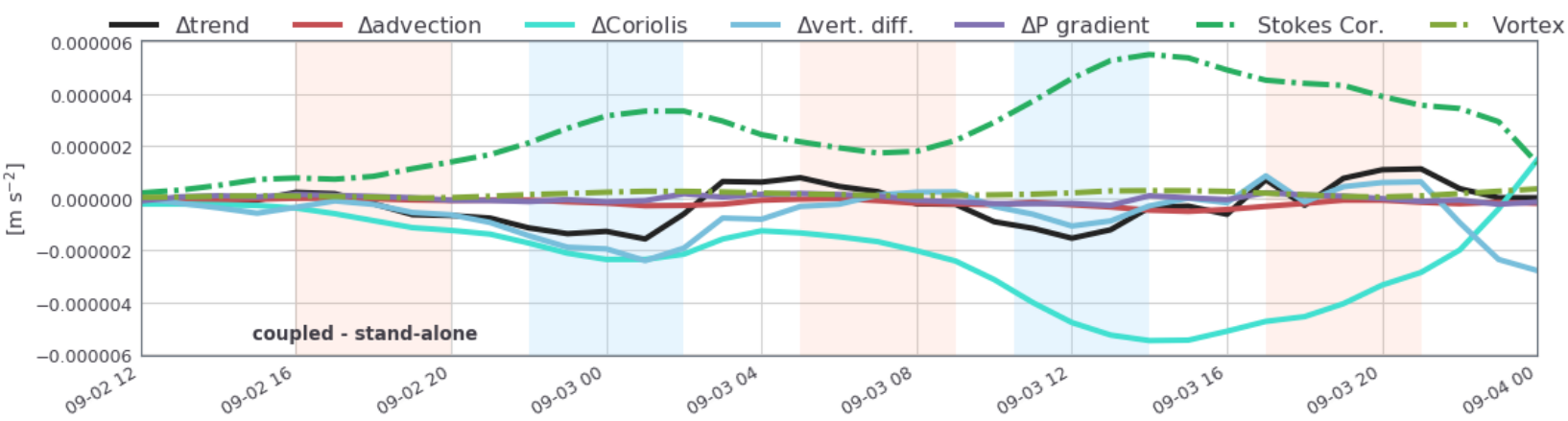

S 2: Time series of (a) the differences in the (quasi-)Eulerian zonal surface current, (b) the terms of the equation for the surface zonal current averaged over the whole domain, and (c) the differences (coupled - stand-alone) of the terms shown in (b). Note that the horizontal diffusion terms and the wave Bernoulli pressure head are omitted as negligible. In (a) the black line represents an average over the whole domain while the blue and red lines are the averages in Z1 (warm, stratified side of front) and Z2 (cold, mixed side of front), respectively. Shadings around the mean curves in (a) correspond to \pm one standard deviation. In (b) the solid lines correspond to the output of the stand-alone run and the dashed lines to the coupled run. Wave related terms are represented by the dot-dashed lines. The light blue and coral shadings demarcate periods of wave-opposing and wave-following currents, respectively. 
(a)

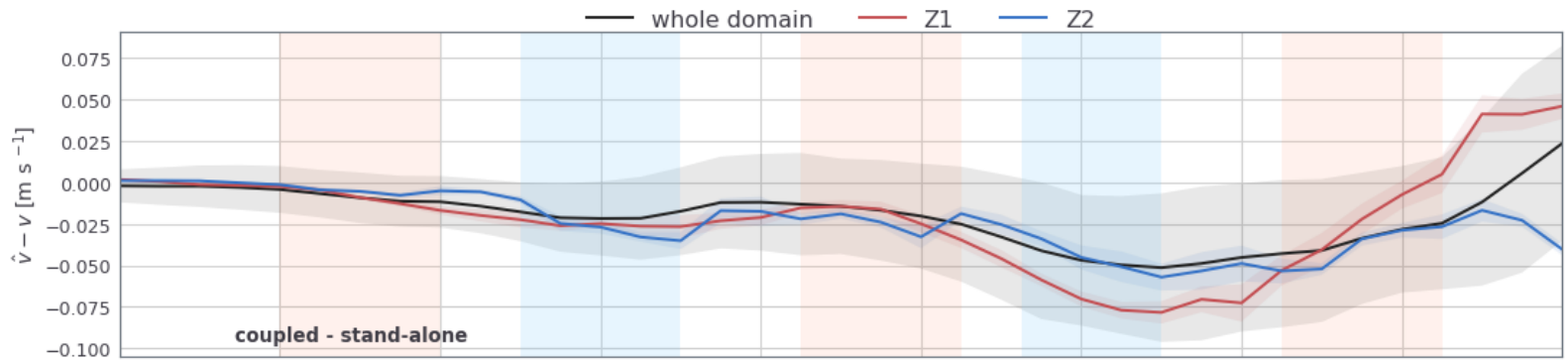

(b)

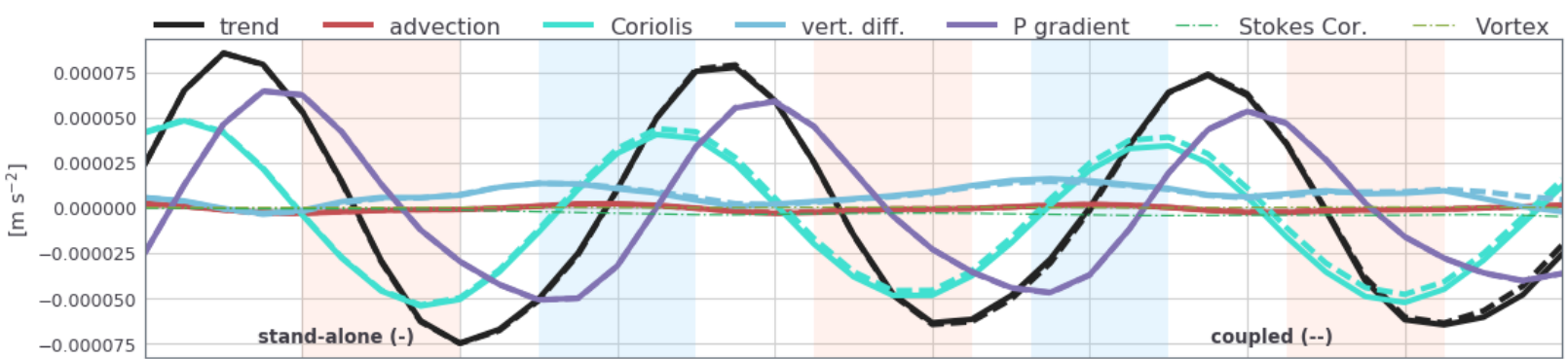

(c)

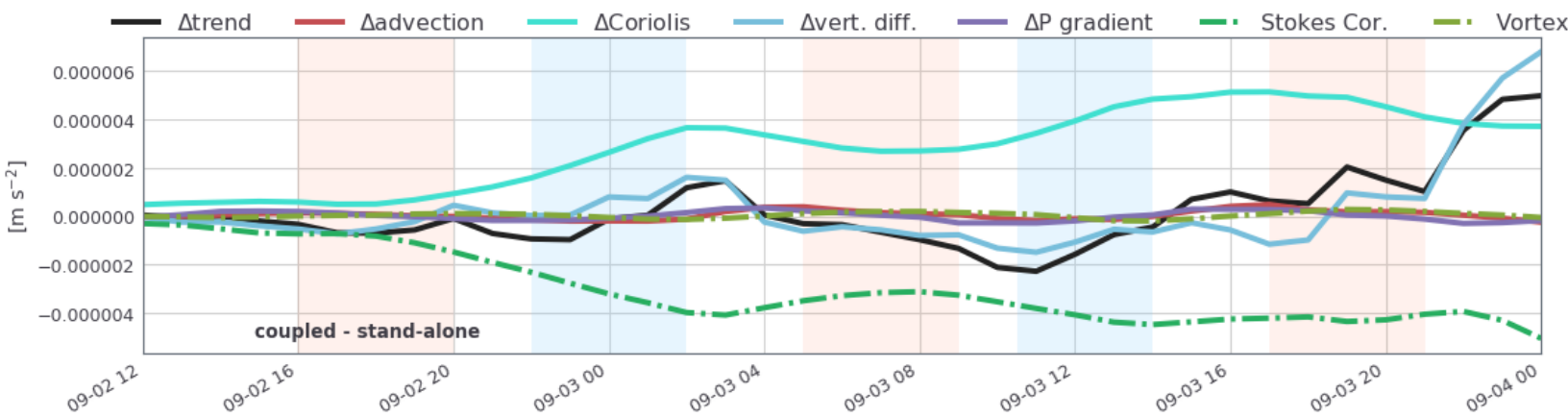

S 3: Time series of (a) the differences in the (quasi-)Eulerian meridional surface current, (b) the terms of the equation for the surface meridional current averaged over the whole domain, and (c) the differences (coupled - stand-alone) of the terms shown in (b). Note that the horizontal diffusion terms and the wave Bernoulli pressure head are omitted as negligible. In (a) the black line represents an average over the whole domain while the blue and red lines are the averages in Z1 (warm, stratified side of front) and Z2 (cold, mixed side of front), respectively. Shadings around the mean curves in (a) correspond to \pm one standard deviation. In (b) the solid lines correspond to the output of the stand-alone run and the dashed lines to the coupled run. Wave related terms are represented by the dot-dashed lines. The light blue and coral shadings demarcate periods of wave-opposing and wave-following currents, respectively. 


\section{References}

Agrawal, Y. C., E. A. Terray, M. A. Donelan, P. A. Hwang, A. Williams III, W. M. Drennan, K. K. Kahma, and S. A. Kitaigorodskii, Enhanced dissipation of kinetic energy beneath surface waves, Nature, 359(6392), 219-20, 1992.

Ardhuin, F., F. Collard, B. Chapron, P. Queffeulou, J.-F. Filipot, and M. Hamon, Spectral wave dissipation based on observations: a global validation, in Proceedings of Chinese-German Joint Symposium on Hydraulics and Ocean Engineering, Darmstadt, Germany, pp. 391-400, iSBN: 3-936146-23-3, 2008.

Ardhuin, F., L. Marié, N. Rascle, P. Forget, and A. Roland, Observation and estimation of lagrangian, stokes, and Eulerian currents induced by wind and waves at the sea surface, Journal of Physical Oceanography, 39(11), 2820-2838, doi:10.1175/2009JPO4169.1, 2009.

Ardhuin, F., N. Rascle, B. Chapron, J. Gula, J. Molemaker, S. T. Gille, D. Menemenlis, and C. Rocha, Small scale currents have large effects on wind wave heights, J. Geophys. Res., 122(C6), 4500-4517, doi:10.1002/2016JC012413, 2017.

Bakker, W. T., and T. Van Doorn, Near-bottom velocities in waves with a current, in Coastal Engineering 1978, pp. 1394-1413, American Society of Civil Engineers, 1978.

Baschek, B., D. M. Farmer, and C. Garrett, Tidal fronts and their role in air-sea gas exchange, Journal of Marine Research, 64(4), 483-515, doi:doi:10.1357/002224006778715766, 2006.

Bennis, A.-C., F. Ardhuin, and F. Dumas, On the coupling of wave and three-dimensional circulation models : Choice of theoretical framework, practical implementation and adiabatic tests, Ocean Modeling, 40, 260-272, 2011.

Bidlot, J., P. Janssen, and S. Abdalla, A revised formulation for ocean wave dissipation in CY25R1, Tech. Rep. Memorandum R60.9/JB/0516, Research Department, ECMWF, Reading, U. K., 2005.

Bolaños, R., J. M. Brown, and A. J. Souza, Wavecurrent interactions in a tide dominated estuary, Continental Shelf Research, 87, 109 - 123, doi:https://doi.org/10.1016/j.csr.2014.05.009, oceanography at coastal scales, 2014.

Boudière, E., C. Maisondieu, F. Ardhuin, M. Accensi, L. Pineau-Guillou, and J. Lepesqueur, A suitable metocean hindcast database for the design of marine energy converters, Int. J. Mar. Energy, 28(3-4), e40-e52, 2013. 
Breivik, ., P. A. E. M. Janssen, and J.-R. Bidlot, Approximate stokes drift profiles in deep water, Journal of Physical Oceanography, 44(9), 2433-2445, doi:10.1175/JPO-D-14-0020.1, 2014.

Burchard, H., Comparing the performance of the mellor-yamada and the $\kappa-\epsilon$ two-equation turbulence models, Journal of Geophysical Research: Oceans, 103(c5), 10,543-10,554, doi:10.1029/98JC00261, 1998.

Burchard, H., Simulating the wave-enhanced layer under breaking surface waves with two-equation turbulence models, J. Phys. Oceanog., 31, 3133-3145, 2001.

Burchard, H., Applied turbulence modelling in marine waters, vol. II, 227 pp., Springer, Berlin, 2002.

Burchard, H., and K. Bolding, Comparative analysis of four second-moment turbulence closure models for the oceanic mixed layer, J. Phys. Oceanog., 31, 1943-1968, 2001.

Canuto, V. M., A. Howard, Y. Cheng, and M. S. Dubovikov, Ocean turbulence. part i: One-point closure modelmomentum and heat vertical diffusivities, Journal of Physical Oceanography, 31 (6), 1413-1426, doi:10.1175/1520-0485(2001)031<1413:OTPIOP>2.0.CO;2, 2001.

Cavaleri, L., B. Fox-Kemper, and M. Hemer, Wind waves in the coupled climate system, Bulletin of the American Meteorological Society, 93(11), 1651-1661, doi:10.1175/BAMS-D-11-00170.1, 2012.

Cavaleri, L., S. Abdalla, A. Benetazzo, L. Bertotti, J.-R. Bidlot, Ø. Breivik, S. Carniel, R. Jensen, J. Portilla-Yandun, W. Rogers, et al., Wave modelling in coastal and inner seas, Progress in oceanography, 167, 164-233, 2018.

Charnock, H., Wind stress on a water surface, Q.J.R. Meteorol. Soc., 81, 639-640, 1955.

Chevallier, C., S. Herbette, L. Marié, P. L. Borgne, A. Marsouin, S. PÃlrã̂l, B. Levier, and C. Reason, Observations of the ushant front displacements with $\mathrm{msg} /$ seviri derived sea surface temperature data, Remote Sensing of Environment, 146, 3 - 10, doi:https://doi.org/10.1016/j.rse.2013.07.038, liege Colloquium Special Issue: Remote sensing of ocean colour, temperature and salinity, 2014.

Craig, P. D., and M. L. Banner, Modeling wave-enhanced turbulence in the ocean surface layer, J. Phys. Oceanogr., 24, 25462559, 1994.

Craik, A. D. D., and S. Leibovich, A rational model for langmuir circulations, J. Fluid Mech., 73, 401426, 1976. 
Debreu, L., and T. Duhaut, Développements numériques pour le modèle mars3d analyse des schémas d'advection verticaux réduction de la diffusion diapycnale, Contrat PREVIMER 09/2 211 097, EPI MOISE, INRIA Rhône-Alpes, Laboratoire Jean Kuntzmann, Grenoble, 2011.

Debreu, L., C. Vouland, and E. Blayo, Agrif: Adaptive grid refinement in fortran, Computers $E_{3}$ Geosciences, 34(1), 8 - 13, doi:https://doi.org/10.1016/j.cageo.2007.01.009, 2008.

Dingemans, M. W., J. A. T. M. van Kester, A. C. Radder, and R. E. Uittenbogaard, The effect of the CL-vortex force in 3D wave-current interaction, in Proceedings of the 25th international conference on coastal engineering, Orlando, pp. 4821-4832, ASCE, 1996.

Dumas, F., and G. Langlois, MARS Model for Applications at Regional Scale Scientific model description, Ifremer, 2009.

Fairall, C. W., E. F. Bradley, J. E. Hare, A. A. Grachev, and J. B. Edson, Bulk parameterization of air-sea fluxes: Updates and verification for the coare algorithm, J. Climate, 16, 571-591, 2003.

Gill, A. E., Atmosphere-Ocean dynamics (International Geophysics Series), academic press, 1982.

Grant, W. D., and O. S. Madsen, Movable bed roughness in unsteady oscillatory flow, J. Geophys. Res., 87(C1), 469-481, 1982.

Groeneweg, J., and J. A. Battjes, Three-dimensional wave effects on a steady current, Journal of Fluid Mechanics, 478, 325-343, 2003.

Harcourt, R. R., A second-moment closure model of langmuir turbulence, Journal of Physical Oceanography, 43(4), 673-697, doi:10.1175/JPO-D-12-0105.1, 2013.

Hasselmann, K., Wave-driven inertial oscillations, Geophys. Fluid Dyn., 1, 463-502, 1970.

Huang, Z., and C. C. Mei, Effects of surface waves on a turbulent current over a smooth or rough seabed, Journal of Fluid Mechanics, 497, 253-287, dOI : 10.1017/S0022112003006657, 2003.

Jones, P. W., First- and second-order conservative remapping schemes for grids in spherical coordinates, Monthly Weather Review, 127(9), 2204-2210, doi:10.1175/15200493(1999)127<2204:FASOCR>2.0.CO;2, 1999.

Kantha, L. H., and C. A. Clayson, On the effect of surface gravity waves on mixing in the oceanic mixed layer, Ocean Modelling, 6, 101-124, 2004. 
Kemp, P., and R. Simons, The interaction of waves and a turbulent current: waves propagating against the current, Journal of fluid mechanics, 130, 73-89, 1983.

Kemp, P. H., and R. R. Simons, The interaction between waves and a turbulent current: waves propagating with the current, Journal of Fluid Mechanics, 116, 227250, doi:10.1017/S0022112082000445, 1982.

Klopman, G., Vertical structure of the flow due to waves and currents, Progress report, Delft Hydraulics H, 840.32, Part I, 1993.

Klopman, G., Vertical structure of the flow due to waves and currents, Progress report, Delft Hydraulics H, 840(Part II), 1994.

Kowalik, Z., and T. S. Murty, Numerical modeling of ocean dynamics, vol. 5, World Scientific, 1993.

Lane, E. M., J. M. Restrepo, and J. C. McWilliams, Wave-current interaction: A comparison of radiationstress and vortex-force representations, Journal of Physical Oceanography, 37, 1122-1141, 2007.

Langmuir, I., Surface motion of water induced by wind, Science, 87, 119-123, 1938.

Lazure, P., and F. Dumas, An external-internal mode coupling for a 3d hydrodynamical model for applications at regional scale (mars), Advances in water resources, 31(2), 233-250, 2008.

Lazure, P., V. Garnier, F. Dumas, C. Herry, and M. Chifflet, Development of a hydrodynamic model of the bay of biscay. validation of hydrology, Continental Shelf Research, 29(8), 985 - 997, doi: https://doi.org/10.1016/j.csr.2008.12.017, 100 Years of Research within the Bay of Biscay, 2009.

Le Boyer, A., G. Cambon, N. Daniault, S. Herbette, B. L. Cann, L. Marié, and P. Morin, Observations of the ushant tidal front in september 2007, Continental Shelf Research, 29(8), in press, doi: 10.1016/j.csr.2008.12.020, 2009.

Leonard, B. P., A. P. Lock, and M. K. MacVean, Conservative explicit unrestricted-time-step multidimensional constancy-preserving advection schemes, Monthly Weather Review, 124(11), 2588-2606, doi:10.1175/1520-0493(1996)124<2588:CEUTSM>2.0.CO;2, 1996.

Lyard, F., F. Lefevre, T. Letellier, and O. Francis, Modelling the global ocean tides: modern insights from fes2004, Ocean dynamics, 56(5-6), 394-415, 2006.

McWilliams, J. C., and B. Fox-Kemper, Oceanic wave-balanced surface fronts and filaments, Journal of Fluid Mechanics, 730, 464490, doi:10.1017/jfm.2013.348, 2013. 
McWilliams, J. C., J. M. Restrepo, and E. M. Lane, An asymptotic theory for the interaction of waves and currents in coastal waters, Journal of Fluid Mechanics, 511, 135-178, 2004.

Olabarrieta, M., R. Medina, and S. Castanedo, Effects of wave-current interaction on the current profile, Coastal Engineering, 57, 643-655, doi:10.1016/j.coastaleng.2010.02.003, 2010.

Osuna, P., and J. Wolf, a numerical study on the effect of wave-current interaction process in the hydrodynamics of the Irish sea, in Proceedings of the 5th International Symposium Ocean Wave Measurement and Analysis, Madrid, june 2005, ASCE, 2005.

Phillips, O. M., On the response of short ocean wave components at a fixed wavenumber to ocean current variations, Journal of Physical Oceanography, 14(9), 1425-33, 1984.

Polton, J. A., D. M. Lewis, and S. E. Belcher, The role of wave-induced Coriolis-Stokes forcing on the wind-driven mixed layer, Journal of Physical Oceanography, 35, 444-457, 2005.

Pringle, K. K., Visual perception by a computer, in Automatic Interpretation and Classification of Images, edited by A.Grasselli, pp. 277-284, Academic Press, New York, 1969.

Ràfols, L., M. Grifoll, and M. Espino, Wave-current interactions in a wind-jet region, Ocean Science, 15(1), 1-20, doi:10.5194/os-15-1-2019, 2019.

Rascle, N., and F. Ardhuin, Drift and mixing under the ocean surface revisited. stratified conditions and model-data comparisons, J. Geophys. Res., 114, C02,016, doi:10.1029/2007JC004466, 2009.

Rascle, N., F. Ardhuin, and E. A. Terray, Drift and mixing under the ocean surface. a coherent onedimensional description with application to unstratified conditions, J. Geophys. Res., 111, C03,016, doi:10.1029/2005JC003004, 2006.

Rascle, N., B. Chapron, F. Ardhuin, and A. Soloviev, A note on the direct injection of turbulence by breaking waves, Ocean Modelling, 70, 145-151, doi:10.1016/j.ocemod.2012.09.001, 2013.

Simpson, J. H., The shelf-sea fronts: implications of their existence and behaviour, Proc. R. Soc. Lond. A, 302, 531, 1981.

Smith, J. A., Wave-current interactions in finite-depth, Journal of Physical Oceanography, 36, 1403-1419, 2006.

Soloviev, A., and R. Lukas, Observation of wave-enhanced turbulence in the near-surface layer of the ocean during TOGA COARE, Deep Sea Research, I 50, 371-395, 2003. 
Song, J.-B., The effects of random surface waves on the steady ekman current solutions, Deep Sea Research Part I: Oceanographic Research Papers, 56(5), 659 - 671, doi:https://doi.org/10.1016/j.dsr.2008.12.014, 2009.

Soulsby, R., Dynamics of marine sands, a manual for practical applications, 256 pp., Thomas Telford Publications, London, 1997.

Soulsby, R. L., L. Hamm, G. Klopman, D. Myrhaug, R. R. Simons, and G. P. Thomas, Wave-current interaction within and outside the bottom boudary layer, Coastal Engineering, 21, 41-69, 1993.

Stopa, J. E., F. Ardhuin, A. Bababin, and S. Zieger, Comparison and validation of physical wave parameterizations in spectral wave models, Ocean Modelling, 103, 2-17, doi:10.1016/j.ocemod.2015.09.003, 2016.

Sullivan, P., and J. McWilliams, Dynamics of winds and currents coupled to surface waves, Annual Review of Fluid Mechanics, 42, 19-42, 2010.

Sullivan, P., J. McWilliams, and W. Melville, The oceanic boundary layer driven by wave breaking with stochastic variability. part 1. direct numerical simulations, J. Fluid Mech., 50\%, doi:10.1017/S0022112004008,882, 2004.

Sullivan, P., J. McWilliams, and W. Melville, Surface gravity wave effects in the oceanic boundary layer: Large-eddy simulation with vortex force and stochastic breakers, J. Fluid Mech., 593, 405452, 2007.

Suzuki, N., On the physical mechanisms of the two-way coupling between a surface wave field and a circulation consisting of a roll and streak, Journal of Fluid Mechanics, 881, 906-950, doi:10.1017/jfm.2019.752, 2019.

Suzuki, N., and B. Fox-Kemper, Understanding stokes forces in the wave-averaged equations, J. Geophys. Res., 121, 3579-3596, doi:10.1002/2015JC011563, 2016.

Suzuki, N., B. Fox-Kemper, P. E. Hamlington, and L. P. van Roekel, Surface waves affect frontogenesis, Geophysical Research Letters, 121, 3597-3624, doi:10.1002/2015JC011563, 2016.

Taylor, G. I., Statistical theory of turbulence., Proceedings of the Royal Society of London. Series AMathematical and Physical Sciences, 151 (873), 465-478, 1935.

Teles, M. J., A. A. Pires-Silva, and M. Benoit, Numerical modelling of wave current interactions at a local scale, Ocean Modelling, 68, 72 - 87, doi:https://doi.org/10.1016/j.ocemod.2013.04.006, 2013. 
Terray, E. A., M. A. Donelan, Y. C. Agrawal, W. M. Drennan, K. K. Kahma, I. A. J. Williams, P. A. Hwang, and S. A. Kitaigorodskii, Estimates of kinetic energy dissipation under surface waves, Journal of Physical Oceanography, 26(5), 792-807, 1996.

Terray, E. A., W. M. Drennan, and M. A. Donelan, The vertical structure of shear and dissipation in the ocean surface layer, in Proc. Symp. on Air-Sea Interaction, Sydney, pp. 239-245, University of New South Wales, 2000.

Tolman, H., The wavewatch iii $($ development group (2014), user manual and system documentation of wavewatch iii $($ version 4.18, Tech. Note 316, NOAA/NWS/NCEP/MMAB, 2014.

Tolman, H. L., M. Accensi, H. Alves, F. Ardhuin, J. Bidlot, N. Booij, A.-C. Bennis, T. Campbell, D. V. Chalikov, A. Chawla, J.-F. Filipot, M. Foreman, P. Janssen, F. Leckler, Jian-Guo, K. L. M. Orzech, R. Padilla-Hernández, W. E. Rogers, A. Rawat, A. Roland, M. D. Sikiric, M. Szyszka, H. L. Tolman, B. Tracy, G. P. van Vledder, A. van der Westhuysen, and S. Zieger, User manual and system documentation of WAVEWATCH-III ${ }^{\mathrm{TM}}$ version 4.18, Tech. Rep. 316, NOAA/NWS/NCEP/MMAB, 2014.

Umeyama, M., Reynolds stresses and velocity distributions in a wave-current coexisting environment, Journal of Waterway, Port, Coastal, and Ocean Engineering, 131(5), 203-212, doi: 10.1061/(ASCE)0733-950X(2005)131:5(203), 2005.

Umlauf, L., A generic length-scale equation for geophysical turbulence models, Journal of marine research, 61(2), 235-265, doi:10.1357/002224003322005087, 2003.

Umlauf, L., and H. Burchard, Second-order turbulence closure models for geophysical boundary layers. a review of recent work, Continental Shelf Research, 25(7), 795 - 827, doi: https://doi.org/10.1016/j.csr.2004.08.004, recent Developments in Physical Oceanographic Modelling: Part II, 2005.

Villas Boâs, A. B., F. Ardhuin, A. Ayet, M. A. Bourassa, P. Brandt, B. Chapron, B. D. Cornuelle, J. T. Farrar, M. R. Fewings, B. Fox-Kemper, S. T. Gille, C. Gommenginger, P. Heimbach, M. C. Hell, Q. Li, M. R. Mazloff, S. T. Merrifield, A. Mouche, M. H. Rio, E. Rodriguez, J. D. Shutler, A. C. Subramanian, E. J. Terrill, M. Tsamados, C. Ubelmann, and E. van Sebille, Integrated observations of global surface winds, currents, and waves: Requirements and challenges for the next decade, Frontiers in Marine Science, 6, 425, doi:10.3389/fmars.2019.00425, 2019.

Voldoire, A., B. Decharme, J. Pianezze, C. Lebeaupin Brossier, F. Sevault, L. Seyfried, V. Garnier, S. Bielli, S. Valcke, A. Alias, M. Accensi, F. Ardhuin, M.-N. Bouin, V. Ducrocq, S. Faroux, H. Giordani, 
F. Léger, P. Marsaleix, R. Rainaud, J.-L. Redelsperger, E. Richard, and S. Riette, Surfex v8.0 interface with oasis3-mct to couple atmosphere with hydrology, ocean, waves and sea-ice models, from coastal to global scales, Geoscientific Model Development, 10(11), 4207-4227, doi:10.5194/gmd-10-4207-2017, 2017.

Walstra, D. J. R., J. Roelvink, and J. Groeneweg, Calculation of wave-driven currents in a 3D mean flow model, in Proceedings of the 27th international conference on coastal engineering, Sydney, vol. 2, pp. 1050-1063, ASCE, 2000.

Wu, L., A. Rutgersson, and E. Sahlée, Upper-ocean mixing due to surface gravity waves, Journal of Geophysical Research: Oceans, pp. 8210 - 8228, doi:10.1002/2015JC011329, 2015.

$\mathrm{Xu}, \mathrm{Z}$, and A. J. Bowen, Wave- and wind-driven flow in water of finite depth, Journal of Physical Oceanography, 24, 1850-1866, 1994.

Yang, S.-Q., S.-K. Tan, S.-Y. Lim, and S.-F. Zhang, Velocity distribution in combined wavecurrent flows, Advances in Water Resources, 29(8), 1196 - 1208, doi:https://doi.org/10.1016/j.advwatres.2005.09.010, 2006.

You, Z.-J., The effect of wave-induced stress on current profiles, Ocean Engineering, 23(7), 619 - 628, doi:https://doi.org/10.1016/0029-8018(95)00058-5, 1996. 